\title{
Finite element modeling of quasi-brittle cracks in 2D and 3D with enhanced strain accuracy
}

\author{
M. Cervera, G. B. Barbat and M. Chiumenti \\ International Center for Numerical Methods in Engineering (CIMNE) \\ Technical University of Catalonia - BarcelonaTECH \\ Edificio C1, Campus Norte, Jordi Girona 1-3 \\ 08034 Barcelona, Spain \\ miguel.cervera@upc.edu,gbarbat@cimne.upc.edu, michele@cimne.upc.edu
}

\begin{abstract}
This paper discusses the finite element modeling of cracking in quasi-brittle materials. The problem is addressed via a mixed strain/displacement finite element formulation and an isotropic damage constitutive model. The proposed mixed formulation is fully general and is applied in 2D and 3D. Also, it is independent of the specific finite element discretization considered; it can be equally used with triangles/tetrahedra, quadrilaterals/hexahedra and prisms.

The feasibility and accuracy of the method is assessed through extensive comparison with experimental evidence. The correlation with the experimental tests shows the capacity of the mixed formulation to reproduce the experimental crack path and the force-displacement curves with remarkable accuracy. Both $2 \mathrm{D}$ and $3 \mathrm{D}$ examples produce results consistent with the documented data.

Aspects related to the discrete solution, such as convergence regarding mesh resolution and mesh bias, as well as other related to the physical model, like structural size effect and the influence of Poisson's ratio, are also investigated.

The enhanced accuracy of the computed strain field leads to accurate results in terms of crack paths, failure mechanisms and force displacement curves. Spurious mesh dependency suffered by both continuous and discontinuous irreducible formulations is avoided by the mixed FE, without the need of auxiliary tracking techniques or other computational schemes that alter the continuum mechanical problem.
\end{abstract}

Keywords: Damage, Cracking, Mixed Finite Elements, Strain Localization, Structural Failure 


\section{Introduction}

Modeling of cracking in quasi-brittle materials has been the object of intensive study in computational solid mechanics over the last five decades. In most of the studies carried out with standard irreducible elements, the attempts to predict the crack path fail because the obtained solution suffers from spurious bias mesh dependency. Several strategies have been developed for dealing with this obstacle.

Cracking problems have traditionally been tackled in two ways: through continuous and discontinuous approaches. In the continuous one, the failure process is modelled by the degradation of the material, at constitutive level. For this so-called smeared crack approach, classical methods were developed by [1-5]. More recently, nonlocal constitutive models [6], gradient enhanced [7, 8] and phase field techniques [8-12] have also been considered within the continuous approach.

In the discontinuous approach, an explicit crack representation is accounted for in the computed geometry and handled as a geometrical discontinuity $[13,14]$. The kinematics of the finite element is enriched to capture the behavior near the propagating crack. Models developed with this approach include, but are not limited to, cohesive interface elements with or without remeshing [15-22], elements with embedded strong discontinuities [23-28], extended finite elements methods [29-34], and meshless and
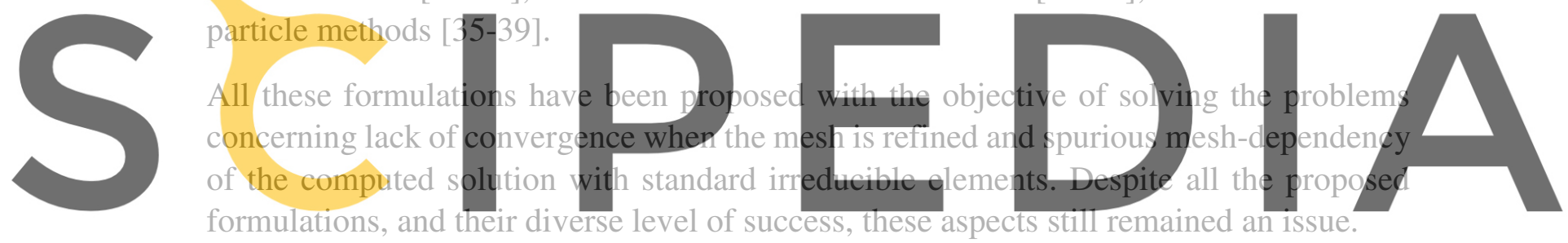

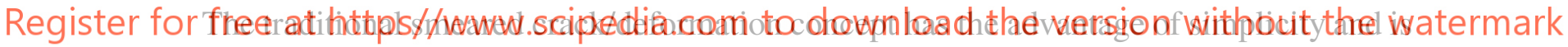
best suited for large-scale analyses. Most efficient from the computational point of view, it is the one favored by commercial FE codes and practitioners. Mesh-size dependency can be solved introducing the fracture energy concept and regularizing with respect the resolution of the FE mesh as proposed by [2].

The continuous approaches that use nonlocal, gradient enhanced or phase field schemes, alter the strong form of the governing equations embedding a length scale related to the width of the localization zone. A clear physical interpretation and direct link between the length parameter in the model and the characteristic length of the material is arguable [40]. An alternative geometrical interpretation has been proposed by [12].

Discontinuous approaches are often regarded as an improvement over the continuous ones, as it is considered that true separation can only be captured with discontinuous techniques. Discontinuous approaches almost invariably require the use of local or global crack tracking auxiliary techniques [41-44]. Those auxiliary techniques do not handle successfully cases that involve complex crack patterns such as multiple branching or intersecting cracks. Besides, they are usually applied only in one type of finite element and lack practical generality, as they require different implementations for each type of finite element. 
In meshless and particle methods [35-39], these drawbacks are avoided through the definition of domains of influence rather than finite elements and the use of appropriate test and trial functions. Local and global remeshing techniques [15-18] also have been used in conjunction with both the continuous and discontinuous approaches.

A comprehensive coverage of all fracture strategies is far beyond the scope of this study that focuses on the application of a mixed FE formulation to the modeling of cracking in quasi-brittle materials. For more details, the reviews in references [45-47] are suggested.

Recently, mixed finite elements have been reexamined by [48-51] to deal with strain localization problems. Mixed finite element formulations have proved to be a remedy for spurious bias mesh dependency, allowing for the computation of strains and stresses with enhanced accuracy both in linear and nonlinear scenarios.

When using the standard finite element formulation, local convergence of the solution in terms of strains cannot be guaranteed in the quasi-singular stress or strain states that occur in the vicinity of the tip of propagating cracks. Even in linear elasticity, local convergence is not guaranteed in quasi-singular points. This lack of local convergence in the strain and stress leads to the spurious mesh bias dependence observed in problems of quasi-brittle crack propagation solved with the standard formulation, yielding incorrect solutions in many cases.
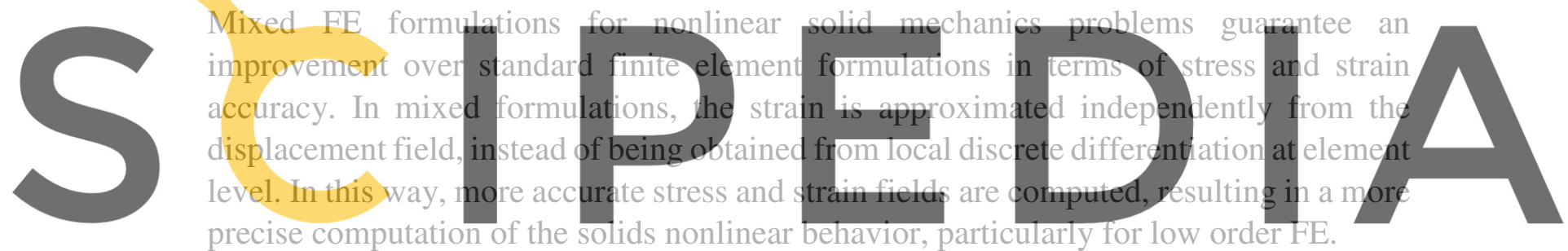

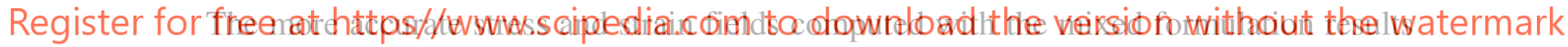
in a significant betterment over the standard formulation, particularly in the prediction of the crack formation and propagation, where mesh-dependence issues are averted. This guarantees convergent results when computing crack trajectories, failure mechanisms and ultimate loads, producing practically mesh-independent solutions using both plasticity and damage constitutive laws.

The leverage of the mixed approach derives from the following strong points:

- It is fully variationally consistent $[49,50]$

- It can be formulated for small or finite displacements or/and kinematics [52, 53]

- It applies equally to 2D and 3D problems [51]

- It is not restricted to a particular FE interpolation, it can be used with triangles/tetrahedra, quadrilaterals/hexahedra or prisms of any order [49-51]

- It is not dependent on the choice of the constitutive equation, it can be applied both for plasticity and/or damage models of any kind [51, 54]

- It can consider isotropic or directional inelastic behavior [55]

- It can address quasi-incompressible situations, including the incompressible limit $[51,56]$

- It can accommodate rate-dependent viscid effects, linear or non-linear 
- It can be extended to include inertial forces in dynamics, or multi-physics phenomena in coupled problems [53]

Also, and regarding cracking problems:

- It follows the classical local constitutive mechanics framework [50]

- It can model Mode I (extension), Mode II (shear) and Mode III (tearing) and mixedmode fracture $[51,55]$

- It can model structural size effect in quasi-brittle failure [54]

- It can accommodate orthotropic damage models with unilateral, crack-closing effects

- It does not require auxiliary crack tracking techniques [49-51]

With reference to the above mentioned alternatives for the computational modeling of quasi-brittle cracks, the mixed finite element formulation here presented fits into the continuous approach, as the crack is represented at constitutive level using a local stress vs. strain relationship. Therefore, the separation between the two opposite sides of the crack is modelled through continuous (linear) displacement and strain fields. No specific degrees of freedom are necessary to model the existing or evolving cracks. Instead, the kinematic enhancement provided by the independent interpolation of displacement and strains proves to be crucial in the numerical solution of strain localization problems.
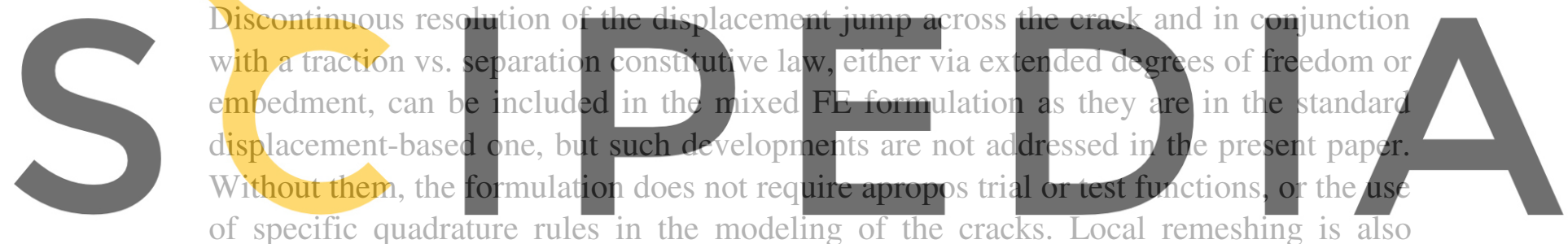
of specific quadrature rules in the modeling of the cracks. Local remeshing is also

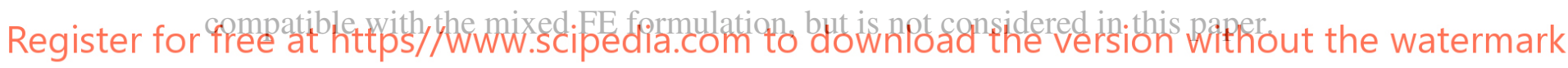

In previous works by the authors, the mixed formulation has been derived and assessed through theoretical benchmark tests and capability demonstration cases, in order to highlight its advantages with regard to the standard form. However, aptness of the proposed model to replicate the behavior of engineering materials observed in experimental tests, specifically related to the crack behavior and propagation, remained open; mixed formulations for computing strain localization has not been adequately validated through correlation with experimental tests.

Therefore, the objectives of this paper are: (1) to present the mixed strain/displacement formulation in matrix notation, ready-to-use for implementation in finite element codes, (2) to demonstrate the application of this format in 2D and 3D applications, (3) to validate the proposed formulation with experimental results. To meet the last two objectives, an extensive comparison with experimental data observed from the literature is performed.

The outline of the paper is as follows. In Section 2, the mixed strain/displacement formulation for the solution of nonlinear solid mechanics problems is presented in matrix form, to be used in conjunction with an isotropic damage model summarized in Section 3. Section 4 presents numerical simulations performed in $2 \mathrm{D}$ and $3 \mathrm{D}$ using the proposed model. The computation results are compared to available data from experimental tests for validation purposes. Finally, conclusions and extensions for future work are presented. 


\section{Mixed strain/displacement formulation}

In the following, the mixed strain-displacement formulation is laid out. Matrix and vector notation based on Voigt's convention for symmetric tensors is adopted, as customarily used in FE literature and in codes.

The formulation of the mixed solid mechanics problem in terms of the stress and displacement fields is classical and it has been used many times in the context of linear elasticity. Mixed FEM have been so derived from the Hellinger-Reissner Variational Principle [57, 58]. However, this is not the most convenient format for the material nonlinear problem. Most of the algorithms used for nonlinear constitutive models in solid mechanics have been derived for the irreducible formulation. This means that these procedures are usually strain driven, and they have a format in which the stress is computed in terms of the strain. Consequently, a mixed FE formulation in terms of the strains and displacement fields as the one used here can incorporate these procedures directly.

\subsection{Variational form}

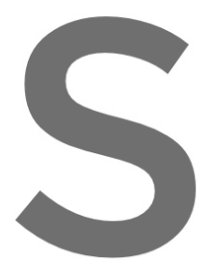

In the following, the variational forn of the
terms of the displacement $\boldsymbol{u}$ and stroin $\varepsilon$ fie
and $\varepsilon$ are expressed in Voigt's convention as

plane strain problems, $u=(u, v)^{T}$ has 2 components and $\varepsilon=\left(\varepsilon_{x}, \varepsilon_{y}, \gamma_{x y}\right)^{T}$ is a 3

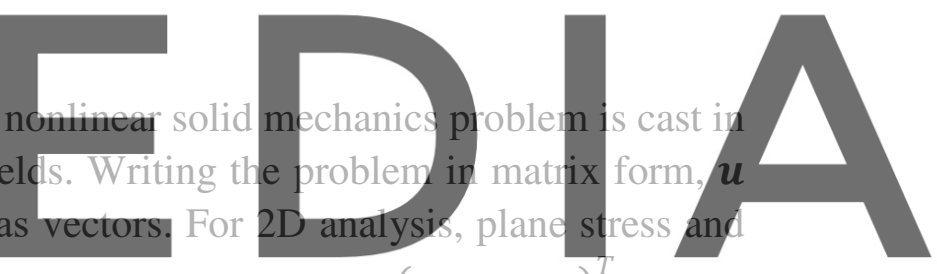

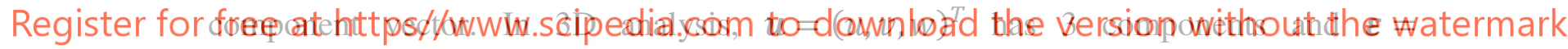

$\left(\varepsilon_{x}, \varepsilon_{y}, \varepsilon_{z}, \gamma_{x y}, \gamma_{y z}, \gamma_{x z}\right)^{T}$ has 6 components [59].

The strain and displacement fields are locally related through the compatibility equation

$$
\varepsilon=\boldsymbol{S u}
$$

where $\mathcal{S}$ is the differential symmetric gradient operator, defined as

$$
\boldsymbol{S}^{T}=\left[\begin{array}{cccccc}
\partial_{x} & 0 & 0 & \partial_{y} & 0 & \partial_{z} \\
0 & \partial_{y} & 0 & \partial_{x} & \partial_{z} & 0 \\
0 & 0 & \partial_{z} & 0 & \partial_{y} & \partial_{x}
\end{array}\right] \quad \text { in } 3 D ; \boldsymbol{S}^{T}=\left[\begin{array}{ccc}
\partial_{x} & 0 & \partial_{y} \\
0 & \partial_{y} & \partial_{x}
\end{array}\right] \text { in } 2 D
$$

where $\boldsymbol{\partial}=\left(\partial_{x}, \partial_{y}, \partial_{z}\right)^{T}$ is the gradient operator in $3 \mathrm{D}$ and $\boldsymbol{\partial}=\left(\partial_{x}, \partial_{y}\right)^{T}$ in 2D.

Correspondingly, the stress $\boldsymbol{\sigma}$ is a vector with 3 components in $2 \mathrm{D}$ analysis, $\boldsymbol{\sigma}=\left(\sigma_{x}, \sigma_{y}, \tau_{x y}\right)^{T}$, and 6 in $3 \mathrm{D}$ analysis, $\boldsymbol{\sigma}=\left(\sigma_{x}, \sigma_{y}, \sigma_{z}, \tau_{x y}, \tau_{y z}, \tau_{x z}\right)^{T}$, whereas the body forces vector $\mathbf{f}$ has 2 in $2 \mathrm{D}, \mathbf{f}=\left(\mathbf{f}_{x}, \mathbf{f}_{y}\right)^{T}$, and 3 components in $3 \mathrm{D}, \mathbf{f}=\left(\mathbf{f}_{x}, \mathbf{f}_{y}, \mathbf{f}_{z}\right)^{T}$. Cauchy's equilibrium equation of a body written in matrix form is 


$$
\boldsymbol{S}^{T} \boldsymbol{\sigma}+\mathbf{f}=\mathbf{0}
$$

where $\boldsymbol{S}^{T}$ is the differential divergence operator, adjoint to the $\boldsymbol{S}$ in (1).

The stress vector $\boldsymbol{\sigma}$ and the strain vector $\boldsymbol{\varepsilon}$ are linked by the constitutive equation:

$$
\boldsymbol{\sigma}=\mathbf{D}_{s} \boldsymbol{\varepsilon}
$$

where $\mathbf{D}_{s}$ is the secant constitutive matrix. For the isotropic damage model laid out in Section 3, the constitutive equation is

$$
\boldsymbol{\sigma}=(1-d) \mathbf{D}_{0} \boldsymbol{\varepsilon}
$$

Pre-multiplying equation (1) by the secant constitutive matrix $\mathbf{D}_{S}$ and introducing equation (4) into equation (3) results in

$$
-\mathrm{D}_{S} \varepsilon+\mathrm{D}_{S} \mathcal{S} u=0
$$
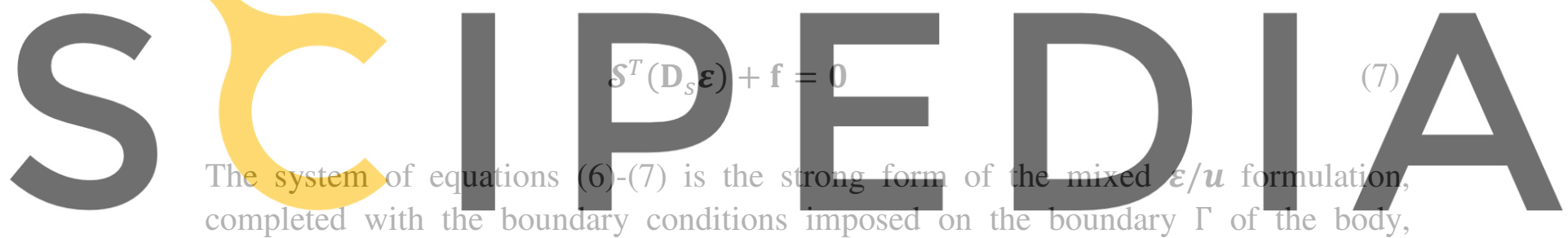

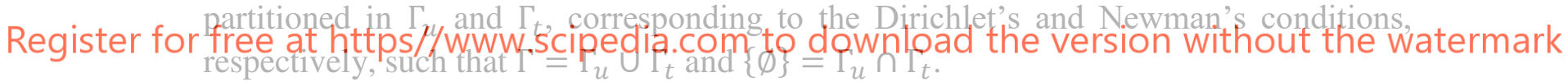

For the sake of conciseness, the prescribed displacements are assumed to vanish on the boundary $\Gamma_{u}$

$$
\boldsymbol{u}=\mathbf{0} \text { in } \Gamma_{u}
$$

The nontrivial case, $\boldsymbol{u}=\overline{\boldsymbol{u}}$ in $\Gamma_{u}$, can be accommodated following standard arguments. Additionally, the prescribed tractions on the boundary $\Gamma_{t}$ are expressed as

$$
\boldsymbol{t}=\overline{\boldsymbol{G}}^{T} \boldsymbol{\sigma}=\overline{\boldsymbol{t}} \text { in } \Gamma_{t}
$$

Where the projection matrix $\overline{\boldsymbol{G}}$ is defined in [59] as

$$
\overline{\boldsymbol{G}}^{T}=\left[\begin{array}{cccccc}
n_{x} & 0 & 0 & n_{y} & 0 & n_{z} \\
0 & n_{y} & 0 & n_{x} & n_{z} & 0 \\
0 & 0 & n_{z} & 0 & n_{y} & n_{z}
\end{array}\right] \text { in } 3 D ; \overline{\boldsymbol{G}}^{T}=\left[\begin{array}{ccc}
n_{x} & 0 & n_{y} \\
0 & n_{y} & n_{x}
\end{array}\right] \text { in } 2 D
$$

where $\boldsymbol{n}=\left(n_{x}, n_{y}, n_{z}\right)^{T}$ is the outward normal vector at the boundary $\Gamma_{t}$. 
The variational form of the problem is obtained as follows.

Firstly, equation (6) is premultiplied by an arbitrary virtual strain vector $\delta \boldsymbol{\varepsilon}$ and integrated over the spatial domain to obtain the weak form of the constitutive and compatibility relationships:

$$
-\int_{\Omega} \delta \boldsymbol{\varepsilon}^{T} \mathbf{D}_{s} \boldsymbol{\varepsilon} d \Omega+\int_{\Omega} \delta \boldsymbol{\varepsilon}^{T} \mathbf{D}_{s} \mathcal{S} \boldsymbol{u} d \Omega=0 \quad \forall \delta \boldsymbol{\varepsilon}
$$

Secondly, equation (7) is premultiplied by an arbitrary virtual displacement vector $\delta \boldsymbol{u}$ and integrated over the spatial domain

$$
\int_{\Omega} \delta \boldsymbol{u}^{T}\left[\boldsymbol{S}^{T}\left(\mathbf{D}_{s} \boldsymbol{\varepsilon}\right)\right] d \Omega+\int_{\Omega} \delta \boldsymbol{u}^{T} \mathbf{f} \mathrm{d} \Omega=0 \quad \forall \delta \boldsymbol{u}
$$

The virtual displacement $\delta \boldsymbol{u}$ complains with the boundary conditions, so that $\delta \boldsymbol{u}=$ 0 in $\Gamma_{u}$. Then, the Divergence Theorem is applied to the first term of equation (12):

\section{$\int_{\Omega} \delta \boldsymbol{u}^{T}\left[\boldsymbol{\delta}^{T}\left(\mathbf{D}_{S} \varepsilon\right)\right] d \Omega=-\int_{\Omega}(\boldsymbol{S} \delta \boldsymbol{u})^{T}\left(\mathbf{D}_{S} \boldsymbol{\varepsilon}\right) d \Omega+\int_{\Gamma} \delta \boldsymbol{u}^{T}\left(\overline{\boldsymbol{G}}^{T} \mathbf{D}_{S} \varepsilon\right) d \Gamma$}

$$
=-\int_{\Omega}(\delta \delta \boldsymbol{u})^{T}\left(\mathbf{D}_{S} \varepsilon\right) d \Omega+\int_{\Gamma_{u}} \delta \boldsymbol{u}^{T}\left(\overline{\boldsymbol{G}}^{T} \mathbf{D}_{s} \varepsilon\right) d \Gamma+\int_{\Gamma_{t}} \delta \boldsymbol{u}^{T} \underbrace{\left(\overline{\boldsymbol{G}}^{T} \mathbf{D}_{s} \varepsilon\right)}_{=\overline{\bar{t}}} d \Gamma
$$
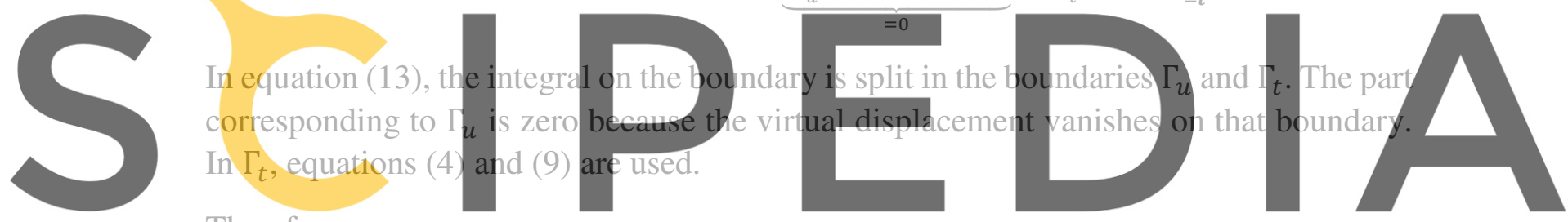

Register for free at https//www.scipedia.com to download the version without the watermark

$$
\int_{\Omega}(\delta \delta \boldsymbol{u})^{T}\left(\mathbb{D}_{S} \varepsilon\right) d \Omega=\int_{\Omega} \delta \boldsymbol{u}^{T} \mathrm{f} \mathrm{d} \Omega+\int_{\Gamma_{t}} \delta \boldsymbol{u}^{T} \bar{t} d \Gamma \quad \forall \delta \boldsymbol{u}
$$

which is the expression of the Virtual Work Principle, as the right hand side term $W(\delta \boldsymbol{u})=\int_{\Omega} \delta \boldsymbol{u}^{T} \mathbf{f} \mathrm{d} \Omega+\int_{\Gamma_{t}} \delta \boldsymbol{u}^{T} \overline{\boldsymbol{t}} d \Gamma$ represents the virtual work done by the tractions $\overline{\boldsymbol{t}}$ and body forces $\mathbf{f}$.

The resulting variational form of the mixed formulation is:

$$
\begin{gathered}
-\int_{\Omega} \delta \boldsymbol{\varepsilon}^{T} \boldsymbol{D}_{s} \boldsymbol{\varepsilon} d \Omega+\int_{\Omega} \delta \boldsymbol{\varepsilon}^{T} \boldsymbol{D}_{s} \delta \boldsymbol{u} d \Omega=0 \quad \forall \delta \boldsymbol{\varepsilon} \\
\int_{\Omega}(\boldsymbol{\delta} \delta \boldsymbol{u})^{T}\left(\mathbf{D}_{s} \boldsymbol{\varepsilon}\right) d \Omega=\int_{\Omega} \delta \boldsymbol{u}^{T} \mathbf{f} \mathrm{d} \Omega+\int_{\Gamma_{t}} \delta \boldsymbol{u}^{T} \overline{\boldsymbol{t}} d \Gamma \quad \forall \delta \boldsymbol{u}
\end{gathered}
$$

The mixed problem to be solved is to find the unknowns $\boldsymbol{u}$ and $\boldsymbol{\varepsilon}$ that verify the system of equations composed by (15) and (16) and that verify the boundary condition $\boldsymbol{u}=\mathbf{0}$ on $\Gamma_{u}$ given the arbitrary virtual displacement $\delta \boldsymbol{u}$, which vanishes on $\Gamma_{u}$ and arbitrary virtual strain $\delta \boldsymbol{\varepsilon}$. Note that this variational problem is symmetric if $\boldsymbol{D}_{s}$ is symmetric. 


\subsection{FE approximation}

At this point, the FE discrete form of the problem is obtained by discretizing the domain in FE, so that $\Omega=\cup \Omega_{e}$, and substituting the displacement $\boldsymbol{u}$ and the strain $\boldsymbol{\varepsilon}$ with the FE discrete approximations $\widehat{\boldsymbol{u}}$ and $\hat{\boldsymbol{\varepsilon}}$ defined element-wise as

$$
\begin{gathered}
\boldsymbol{u} \cong \widehat{\boldsymbol{u}}=\boldsymbol{N}_{u} \boldsymbol{U} \\
\boldsymbol{\varepsilon} \cong \widehat{\boldsymbol{\varepsilon}}=\boldsymbol{N}_{\varepsilon} \boldsymbol{E}
\end{gathered}
$$

where $\boldsymbol{U}$ and $\mathbf{E}$ are vectors containing the values of the displacements and the strains at the nodes of the finite element mesh. $\boldsymbol{N}_{u}$ and $\boldsymbol{N}_{\varepsilon}$ are the matrices containing the interpolation functions adopted in the FE approximation.

In the Galerkin method, the same approximation is considered for the discrete virtual displacements and virtual strains so that

$$
\delta \boldsymbol{u} \cong \delta \widehat{\boldsymbol{u}}=N_{u} \delta U
$$
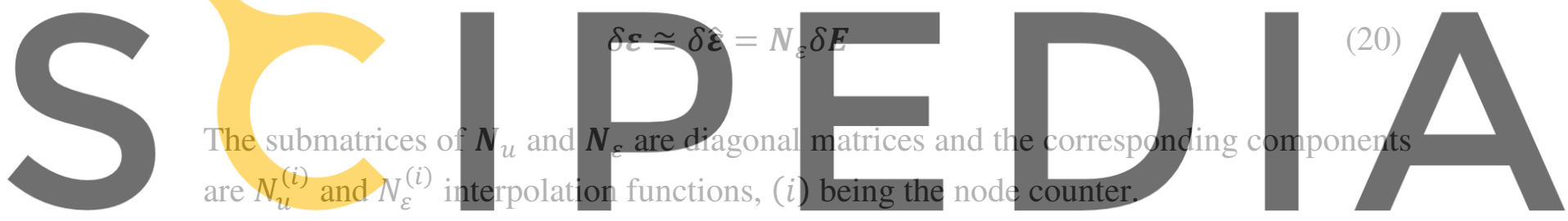

Introducing these approximations, equations (15) and (16) now become:

Register for free at https//wwW.scipedia.com to download the version without the watermark

$$
\begin{gathered}
-\int_{\Omega} \delta E^{T} N_{\varepsilon}^{T} \mathbb{D}_{S} N_{\varepsilon} E d \Omega+\int_{\Omega} \delta E^{T} N_{\varepsilon}^{T} \mathbb{D}_{S} \underbrace{S N_{u}}_{=\boldsymbol{B}_{u}} U d \Omega=0 \quad \forall \delta \boldsymbol{E} \\
\int_{\Omega} \underbrace{\left(\boldsymbol{S} \boldsymbol{N}_{\boldsymbol{u}} \delta \boldsymbol{U}\right)^{T}}_{=\delta \boldsymbol{U}^{T}\left(\boldsymbol{\delta} \boldsymbol{N}_{u}\right)^{T}=\delta \boldsymbol{U}^{T} \boldsymbol{B}_{u}^{T}}\left(\boldsymbol{D}_{s} \boldsymbol{N}_{\boldsymbol{\varepsilon}} \boldsymbol{E}\right) d \Omega=\widehat{W}(\delta \boldsymbol{U}) \quad \forall \delta \boldsymbol{U}
\end{gathered}
$$

where $\boldsymbol{B}_{u}$ is the discrete strain-displacement matrix defined as

$$
\boldsymbol{B}_{u}=\boldsymbol{S} \boldsymbol{N}_{u}
$$

The submatrices of $\boldsymbol{B}_{u}$ have the structure corresponding to the $\boldsymbol{S}$ operator in equation (2), and their components are the Cartesian derivatives of the $N_{u}^{(i)}$ and $N_{\varepsilon}^{(i)}$ interpolation functions $\left(\frac{\partial N^{(i)}}{\partial x} ; \frac{\partial N^{(i)}}{\partial y} ; \frac{\partial N^{(i)}}{\partial z}\right),(i)$ being the node counter.

In (22), $\widehat{W}(\delta \boldsymbol{U})$ is the work done by the tractions $\overline{\boldsymbol{t}}$ and body forces $\mathbf{f}$ defined as 


$$
\widehat{W}(\delta \boldsymbol{U})=\int_{\Omega} \delta \boldsymbol{U}^{T} \boldsymbol{N}_{u}^{T} \mathbf{f} d \Omega+\int_{\Gamma_{t}} \delta \boldsymbol{U}^{T} \boldsymbol{N}_{u}^{T} \overline{\boldsymbol{t}} d \Gamma
$$

In (21) and (22) and henceforth, integrals over the domain are understood as the sum of the integrals over the elements in the FE mesh

$$
\int_{\Omega}(\cdot) d \Omega=\sum_{e} \int_{\Omega_{e}}(\cdot) d \Omega_{e}
$$

Also, with some abuse of notation, $\boldsymbol{U}$ and $\boldsymbol{E}$ (and $\boldsymbol{\delta} \boldsymbol{U}$ are $\boldsymbol{\delta} \boldsymbol{E}$ ) are to be interpreted as the nodal values over the whole FE mesh. This implies the corresponding assembling operations for elemental matrices and vectors into global entities.

Note again that, if matrix $\boldsymbol{D}_{s}$ is symmetric, the discrete system (21)-(22) is symmetric but indefinite.

\section{The virtual displacement $\delta \boldsymbol{U}$ and virtual strain $\delta \boldsymbol{E}$ nodal vectors that appear in equations (21)-(22) are arbitrary. Therefore, the system of equations for the mixed Galerkin method} becomes
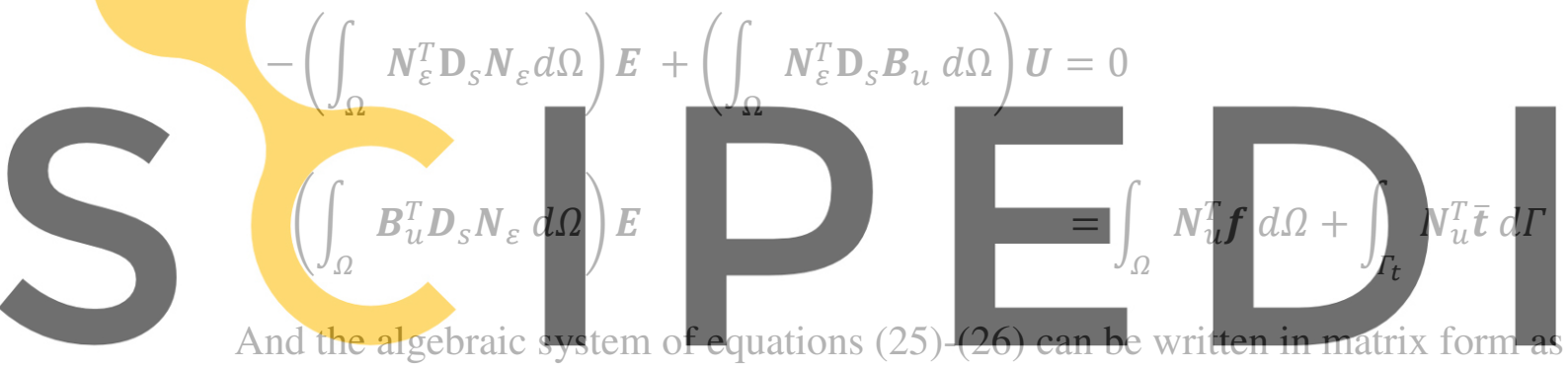

And the algebraic yystem of equations (25)-

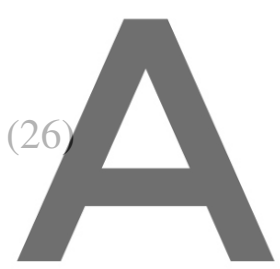

Register for free at https//www.scipedia.comEto download the version without the watermark

where $\left[\begin{array}{ll}\boldsymbol{E} & \boldsymbol{U}\end{array}\right]^{T}$ is the array of nodal values of strains and displacements and

$$
\begin{gathered}
\boldsymbol{M}=\int_{\Omega} \boldsymbol{N}_{\varepsilon}^{T} \mathbf{D}_{s} \boldsymbol{N}_{\varepsilon} d \Omega \\
\boldsymbol{G}=\int_{\Omega} \boldsymbol{N}_{\varepsilon}^{T} \mathbf{D}_{s} \boldsymbol{B}_{u} d \Omega \\
\boldsymbol{F}=\int_{\Omega} \boldsymbol{N}_{u}^{T} \boldsymbol{f} d \Omega+\int_{\Gamma_{t}} \boldsymbol{N}_{u}^{T} \overline{\boldsymbol{t}} d \Gamma
\end{gathered}
$$

$\boldsymbol{M}$ is a mass like projection matrix, $\boldsymbol{G}$ is the discrete gradient matrix and $\boldsymbol{F}$ is the vector of external nodal forces.

In the system (27), the nodal values $\boldsymbol{E}$ can be formally eliminated to write the solution in terms of the nodal displacements $\boldsymbol{U}$ only, as follows. From the first equation in (27), the nodal values for the strains $\boldsymbol{E}$ can be obtained as 


$$
E=M^{-1} G U
$$

which can be substituted into the second equation to yield

$$
\boldsymbol{U}=\left(\boldsymbol{G}^{T} \boldsymbol{M}^{-\mathbf{1}} \boldsymbol{G}\right)^{-\mathbf{1}} \boldsymbol{F}
$$

where $\left(\boldsymbol{G}^{T} \boldsymbol{M}^{-\mathbf{1}} \boldsymbol{G}\right)^{-\mathbf{1}}$ is the Schur complement of $-\boldsymbol{M}$ in the system (27).

\subsection{VMS stabilization}

To ensure solvability (i.e. uniqueness) and stability of the solution in the algebraic system of equations (27), the interpolation functions in (17)-(18) must satisfy the Inf-Sup condition [60-62]. This condition is not verified if equal interpolations are used for strains and displacements. In that case, the solution is unstable, and uncontrollably spurious oscillations may appear in the computed displacement field. To be able to circumvent the strictness of the Inf-Sup condition and to use linear approximations in both interpolation functions, a stabilization procedure is necessary to provide the necessary stability to the mixed discrete formulation. The stabilization procedure consists in the modification of the discrete variational form using the Orthogonal Subscales Method, introduced in [63] within the framewor adopted herein.

The basic idea of the
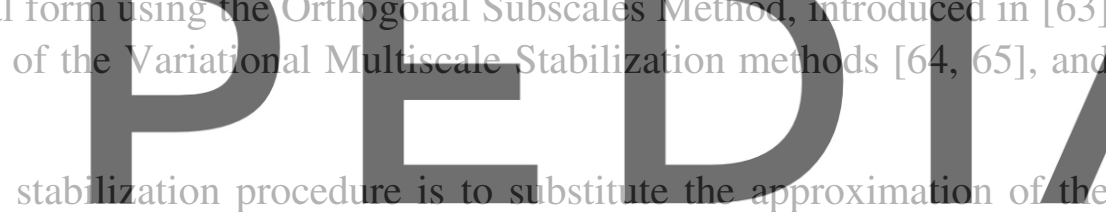

discrete strain in equation (18) by the following stabilized discrete field

Register for free at https//www.scipedia.com to download the version without the watermark

$$
\varepsilon \cong \widehat{\varepsilon}=N_{\varepsilon} E+\tau_{\varepsilon}\left(B_{u} U-N_{\varepsilon} E\right)=\left(1-\tau_{\varepsilon}\right) N_{\varepsilon} E+\tau_{\varepsilon} B_{u} U
$$

where $\tau_{\varepsilon}$ is a stabilization parameter with value $0 \leq \tau_{\varepsilon} \leq 1$. Note that for $\tau_{\varepsilon}=1$, the strain interpolation of the standard irreducible formulation is recovered:

$$
\boldsymbol{\varepsilon} \cong \widehat{\boldsymbol{\varepsilon}}=\boldsymbol{B}_{u} \boldsymbol{U}
$$

Making the corresponding substitution in equations (15) and (16), the final stabilized set of mixed FE equations is:

$$
\begin{aligned}
-\left(1-\tau_{\varepsilon}\right) \int_{\Omega} \boldsymbol{\delta} \boldsymbol{E}^{T} \boldsymbol{N}_{\varepsilon}^{T} \mathbf{D}_{s} \boldsymbol{N}_{\varepsilon} \boldsymbol{E} d \Omega+\left(1-\tau_{\varepsilon}\right) \int_{\Omega} \boldsymbol{\delta} \boldsymbol{E}^{T} \boldsymbol{N}_{\varepsilon}^{T} \mathbf{D}_{s} \boldsymbol{B}_{u} \boldsymbol{U} d \Omega & =0 \quad \forall \delta \boldsymbol{E} \\
\left(1-\tau_{\varepsilon}\right) \int_{\Omega} \delta \boldsymbol{U}^{T} \boldsymbol{B}_{u}^{T}\left(\boldsymbol{D}_{s} \boldsymbol{N}_{\varepsilon} \boldsymbol{E}\right) d \Omega+\tau_{\varepsilon} \int_{\Omega} \delta \boldsymbol{U}^{T} \boldsymbol{B}_{u}^{T} \boldsymbol{D}_{s} \boldsymbol{B}_{u} \boldsymbol{U} d \Omega & =\widehat{W}(\delta \boldsymbol{U})
\end{aligned}
$$


The stabilization used is variationally consistent: converging values of the unknowns $\boldsymbol{\varepsilon}$ and $\boldsymbol{u}$ which satisfy the Galerkin system (21)-(22) also satisfy the stabilized form (35)(36). This is because residual-based stabilization procedures do not introduce any additional approximation nor any consistency error. For a converged solution, when the size of the element $h$ tends to zero, $h \rightarrow 0, \boldsymbol{\varepsilon} \rightarrow \boldsymbol{N}_{\varepsilon} \boldsymbol{E}=\boldsymbol{B}_{u} \boldsymbol{U}$ and the stabilization term vanishes. For non-converged situation, the added terms $\tau_{\varepsilon}\left(\boldsymbol{B}_{u} \boldsymbol{U}-\boldsymbol{N}_{\varepsilon} \boldsymbol{E}\right)$ are small, as they depend on the difference between two approximations of different order to the same quantity.

Therefore, for a given FE mesh, using different values of the stabilization procedure yields slightly different results. However, the consistency of the residual-based stabilization guarantees convergence to the unique solution. Using different stabilization parameters on the same mesh is akin to use different FE interpolations of the same order of convergence with the same nodal arrangement.

Moreover, note that optimal convergence rate in linear problems is obtained reducing the stabilization on mesh refinement [49], such that

$$
\tau_{\varepsilon}=c_{\varepsilon} \frac{h}{L_{0}}
$$

where $c_{\varepsilon}$ is an arbitrary positive numbers, $h$ is the finite element size and $L_{0}$ is the characteristic size of the problem. In nonlinear problems involving damage, the stabilization parameter is affected by the reduction of stiffness in the damaged elements, so that

$$
\tau_{\varepsilon}=(1-d) c_{\varepsilon} \frac{h}{L_{0}}
$$

When quasi-incompressible situations need to be modelled, additional consistent stabilization terms, equally based on residual considerations at discrete level, need to be added [51].

The stabilized system of equations is

$$
\left[\begin{array}{cc}
-M_{\tau} & G_{\tau} \\
G_{\tau}^{T} & K_{\tau}
\end{array}\right]\left[\begin{array}{l}
E \\
U
\end{array}\right]=\left[\begin{array}{l}
\mathbf{0} \\
F
\end{array}\right]
$$

with $\boldsymbol{M}_{\boldsymbol{\tau}}=\left(1-\tau_{\varepsilon}\right) \boldsymbol{M}, \boldsymbol{G}_{\boldsymbol{\tau}}=\left(1-\tau_{\varepsilon}\right) \boldsymbol{G}$ and $\boldsymbol{K}_{\boldsymbol{\tau}}=\tau_{\varepsilon} \boldsymbol{K}$ with

$$
\boldsymbol{K}=\int_{\Omega} \boldsymbol{B}_{u}^{T} \boldsymbol{D}_{s} \boldsymbol{B}_{u} d \Omega
$$

In the stabilized system (37), the nodal values $\boldsymbol{U}$ can be formally computed as

$$
\boldsymbol{U}=\left(\boldsymbol{G}_{\boldsymbol{\tau}}^{T} \boldsymbol{M}_{\boldsymbol{\tau}}^{-1} \boldsymbol{G}_{\boldsymbol{\tau}}+\boldsymbol{K}_{\boldsymbol{\tau}}\right)^{-1} \boldsymbol{F}
$$


where, now, the stabilization ensures definitiveness, uniqueness and stability of the solution if $\boldsymbol{K}$ is positive definite. Note again that for $\tau_{\varepsilon}=1$, the stable solution of the standard form $\boldsymbol{U}=\boldsymbol{K}^{-\mathbf{1}} \boldsymbol{F}$ is recovered.

The discrete approximation in equation (33) is not to be interpreted point-wise, as in the VMS method only the variational effect of the stabilization is sought for. This means that the internal variables and secant matrix of the constitutive equations can be computed according to either of the alternative, discrete strain approximation (20) or (33).

\subsection{Implementation and computational aspects}

Nonlinear constitutive behavior such as the one considered in this work (see Section 3) requires an iterative procedure for solving the resulting nonlinear system of equations. In the present work, an iterative Picard's secant algorithm has been used. The problem is solved incrementally in a (pseudo) time step-by-step manner, solving the nonlinear system of equations at each step. Convergence at each time step is achieved when the ratio between the norm of residual forces and the norm of total external forces is lower than a certain imposed tolerance. Some of the analysis were performed under CMOD (crack mouth opening displacement) control in order to capture the complete post-peak behavior.

\section{Isotropic damage model}

For the evaluation of the stresses from the strains and the evaluation of the secant constitutive matrix $\boldsymbol{D}_{s}$, in equations (4) and (5), an isotropic damage model is used. The model adopted here, suitable for concrete, defines the effective equivalent stress through the Rankine and the Drucker-Prager criterions.

From equations (4) and (5)

$$
\boldsymbol{D}_{s}=(1-d) \boldsymbol{D}_{0}
$$

where $d$ is the internal damage index and $\boldsymbol{D}_{0}$ is the elastic constitutive matrix. The damage index $d$ is an internal variable that measures the loss of stiffness of the material and it ranges $0 \leq d \leq 1$.

For the computation of the evolution of the internal damage index, the effective stress $\overline{\boldsymbol{\sigma}}$ is defined as $\overline{\boldsymbol{\sigma}}=\boldsymbol{D}_{0} \boldsymbol{\varepsilon}$. The corresponding equivalent effective stress $\sigma_{e q}$ is defined through the damage criterion, $\sigma_{e q}=F(\overline{\boldsymbol{\sigma}})$. Tensile damage is modelled according to Rankine's criterion, so that

$$
\sigma_{e q}=F(\bar{\sigma})=\left\langle\bar{\sigma}_{1}\right\rangle
$$


where $\bar{\sigma}_{1}$ is the major principal effective stress and $\langle\cdot\rangle$ are the Macaulay brackets, such that $\langle x\rangle=x$ if $x \geq 0, \quad 0$ if $x<0$. For mixed loading, a Drucker-Prager criterion is used, so that

$$
\sigma_{e q}=F(\overline{\boldsymbol{\sigma}})=\frac{3}{3+\tan \phi}\left(\sqrt{3 J_{2}}+\tan \phi \frac{I_{1}}{3}\right)
$$

When $I_{1}$ and $J_{2}$ are the first and second effective stress invariants and $\phi$ is the internal friction angle of the material; this can be related to the uniaxial tensile and compressive strengths, $f_{t}$ and $f_{c}$, as

$$
\tan \phi=3 \frac{f_{c}-f_{t}}{f_{c}+f_{t}}
$$

The damage criterion, $\mathbb{F}$, is defined as

$$
\mathbb{F}=\sigma_{e q}-r(\bar{\sigma}) \leq 0
$$

where $r$ is the current stress-like damage threshold. Its initial value is the tensile strength of the material, $r_{0}=f_{t}$. The current value of the damage threshold is explicitly updated as

$$
r=\max \left(r_{0}, \max \sigma_{e q}(\hat{t})\right) \quad \hat{t} \in[0, t]
$$

This follows from the Kuhn-Tucker optimality and consistency conditions. It guarantees the irreversibility of damage and the positiveness of the dissipation.

The evolution of the internal damage variable is defined by

$$
d=d(r)=1-\frac{r_{0}}{r} \exp \left(-2 H_{S}\left(\frac{\left\langle r-r_{0}\right\rangle}{r_{0}}\right)\right)
$$

where $H_{S}$ is the positive softening parameter, which controls the rate of material degradation.

In FE simulations of quasi-brittle failure, the softening parameter is linked to the material fracture energy $G_{f}$, which is a property of the material, in the following way:

$$
H_{S}=\frac{\bar{H}_{S} b}{1-\bar{H}_{S} b}
$$

where $b$ is the bandwidth of the smeared crack and $\bar{H}_{S}$ is

$$
\bar{H}_{S}=\frac{\left(f_{t}\right)^{2}}{2 E G_{f}}
$$

$f_{t}$ being the tensile strength and $E$ the Young's modulus. In this work 


$$
b=\left(1-\tau_{\varepsilon}\right) 2 h+\tau_{\varepsilon} h=\left(2-\tau_{\varepsilon}\right) h
$$

where $h$ is the finite element size. This is coherent with the approximation adopted for the discrete strain field in equation (33).

\section{Numerical simulations}

In this section, six numerical simulations are performed using the mixed strain/displacement FE formulation laid out in the aforesaid. The numerical solutions are compared with the results of experimental tests reported in the literature. The simulations are:

1. An L-shaped panel subjected to a vertical load

2. A wedge-splitting test

3. Two mixed mode bending beam tests

4. Three notched beams with holes

5. Four-point bending test on a doubly-notched beam

6. Non-planar crack on a three-point bending test on skew notched beam

The examples have been solved using both 2D and 3D finite elements, using triangles or quadrilaterals for 2D and tetrahedra, hexahedra or prisms for the 3D simulations. All the problems are studied by means of a smeared crack approach. No tracking technique is used in any of the cases.

For this, calculations are performed with an enhanced version of the finite element program COMET [66]. Pre- and post-processing are done with GiD [67], developed at CIMNE (International Center for Numerical Methods in Engineering).

\subsection{L-shaped panel}

The numerical analysis of a concrete L-shaped panel subjected to vertical load is considered; corresponding experiments are reported in [68]. Other numerical solutions are reported in [41] and [42]. Reference [41] used embedded crack methods and crack tracking auxiliary techniques while reference [42] used extended finite elements for making their computations.

The geometry and loading is shown in Figure 1 and the material parameters are given in Table 1. The thickness of the panel is $0.1 \mathrm{~m}$.

The load $\boldsymbol{F}$ is applied via increments of vertical displacement at the top left corner of the panel. 
Table 1. Material parameters of the L-shaped panel

\begin{tabular}{|c|c|}
\hline Young's Modulus & $25.85 \cdot 10^{9} \mathrm{~Pa}$ \\
\hline Poisson's Ratio & 0.18 \\
\hline Tensile Strength & $2.7 \cdot 10^{6} \mathrm{~Pa}$ \\
\hline Fracture Energy & $160 \mathrm{~J} / \mathrm{m}^{2}$ \\
\hline
\end{tabular}

This example is solved with the mixed FEM using 2D quadrilateral and 3D hexahedral elements. The computational domain is discretized with fully structured meshes with elements of $8.33 \mathrm{~mm}$, resulting in a mesh of 11,041 nodes for the 2D analysis and 44,164 nodes for the 3D analysis, shown in Figure 2. The 3D mesh is obtained by the out-ofplane extrusion of the 2D mesh. For the 2D analysis, plane stress conditions are assumed.

Figure 3 shows the computed tensile damage contour fills for an imposed vertical displacement of $1 \mathrm{~mm}$ obtained in the 2D and 3D analyses. Both results are identical, as the same mesh configuration is used in the XY plane of the panel in the $2 \mathrm{D}$ and $3 \mathrm{D}$ cases.

In the present FE formulation, the separation between the two opposite sides of the crack is modelled through continuous (linear) displacement and strain fields and the crack is accordingly smeared. The crack surface in the 3D analysis can be depicted as in Figure 4 , plotted as an iso-level surface of the norm of displacements. It corresponds exactly with the crack path obtained in the $2 \mathrm{D}$ analysis.

These results are within the experimental range obtained by [68], as can be observed in Figure 5. In the FE-simulation, the crack propagates as expected from the experimental tests; no spurious mesh bias is observed, although no auxiliary local or global crack tracking techniques is used, nor any initial notch or flaw is imposed in the geometry of the panel to assure the correct crack path at the early stages of the crack formation, contrary to [41] and [42].

Figure 6 shows the computed load-imposed vertical displacement curve obtained in the 2D and 3D simulations, compared to the results from the tests in [68]. As shown, the numerical curves are almost overlapping, demonstrating the closeness of plane stress assumption in $2 \mathrm{D}$. The results are inside the experimental range observed in the tests. It can be seen that the peak is accurately reproduced, as well as the general behavior of the curve.

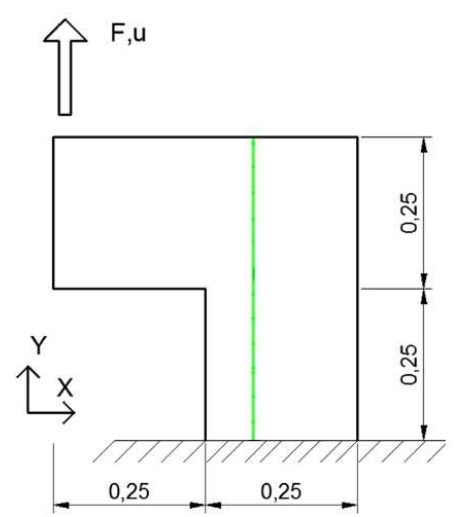

Figure 1. Geometry of the L-shaped panel (m) and vertical axis considered for line graphs 


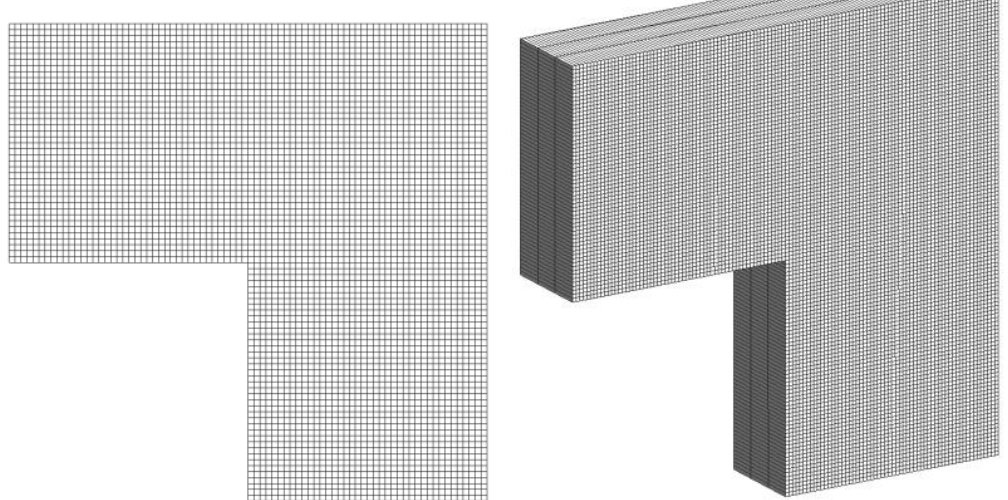

Figure 2. 2D and 3D meshes used for the analysis of the L-shaped panel
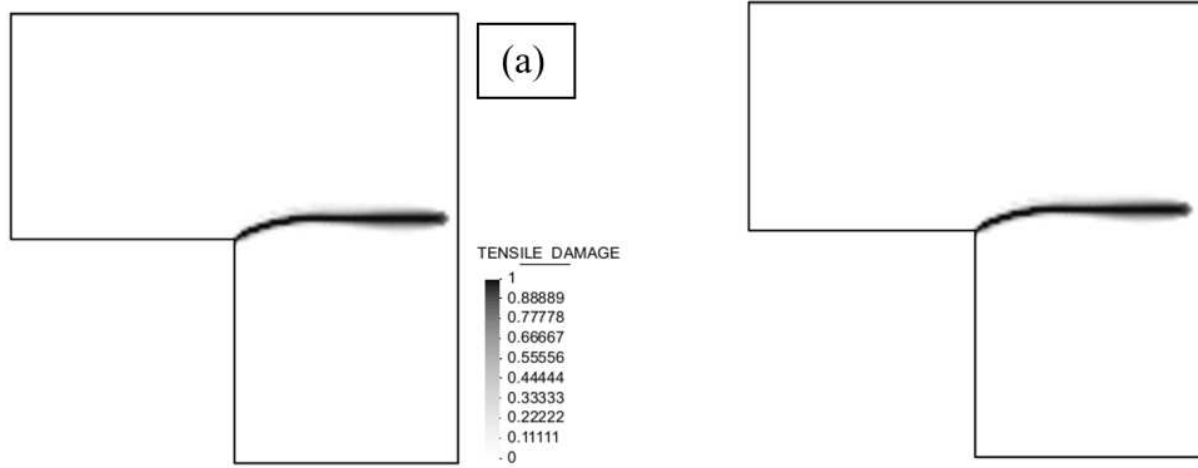

(b)

Figure 3. Tensile damage contour fills for the L-shaped panel, (a) 2D and (b) 3D solution

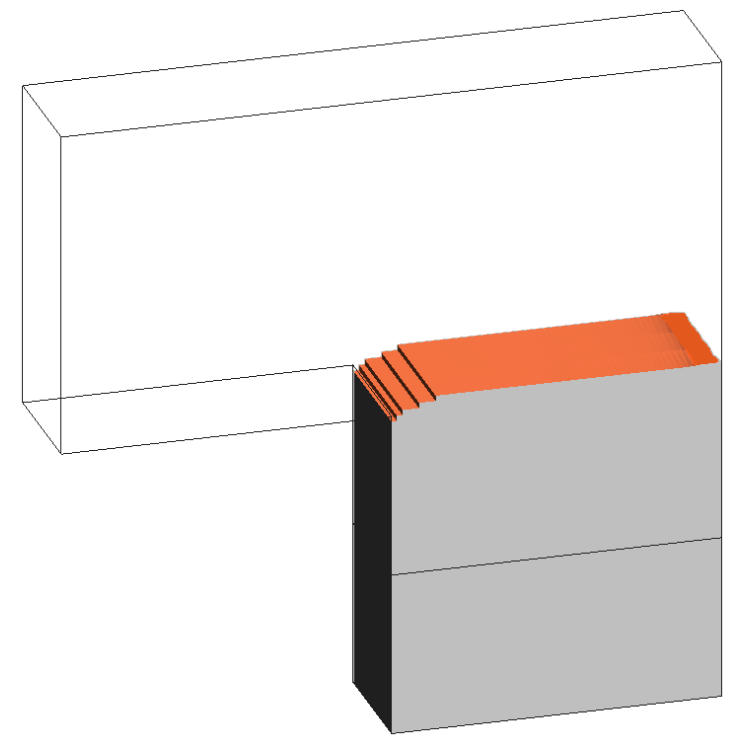

Figure 4. Crack Surface of the L-shaped panel 


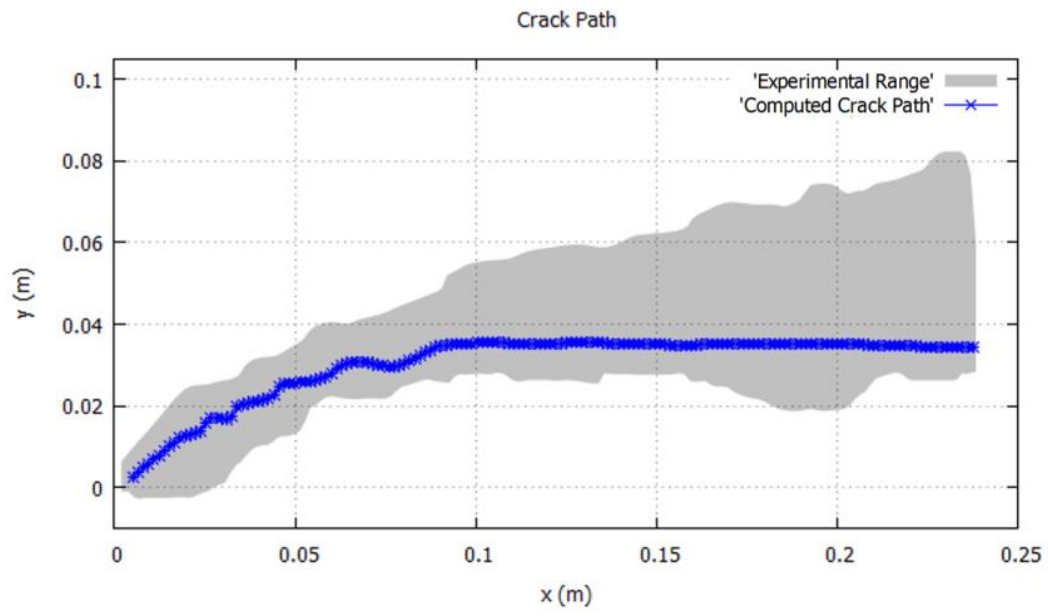

Figure 5. Crack path in the L-shaped panel compared to the experimental range reported in [68]

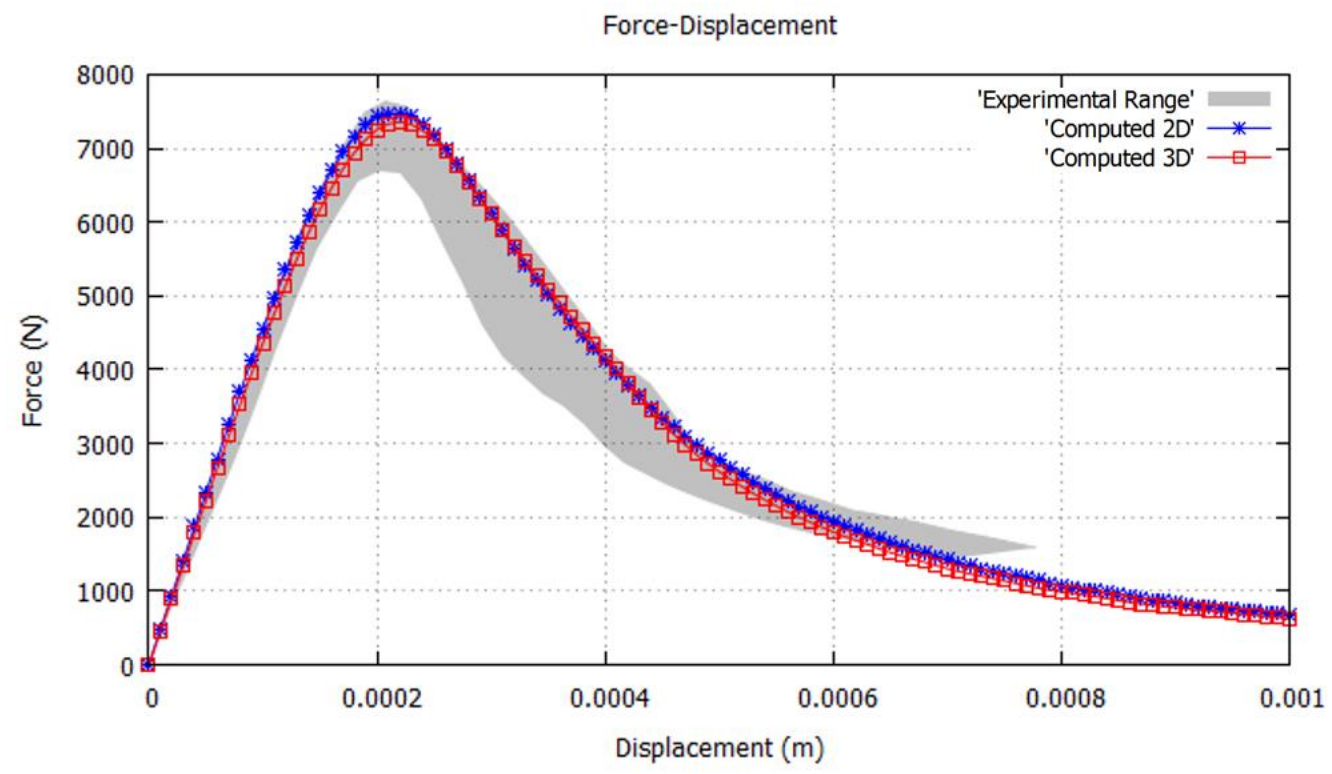

Figure 6. Force-displacement results for 2D and 3D analyses of the L-shaped panel

\section{Independence of results with $\mathrm{FE}$ size}

Thanks to the regularization procedure introduced in equation (49), the computed structural response is independent from the resolution of the FE mesh. This is shown in Figure 7, where the Force-Displacement curve obtained from grids of different sizes $(h=$ $4,8,12 \mathrm{~mm})$ are compared. The results obtained in the $3 \mathrm{D}$ analysis $(h=8 \mathrm{~mm})$ are also shown. All the results are practically overlapping, demonstrating mesh-size independence. 


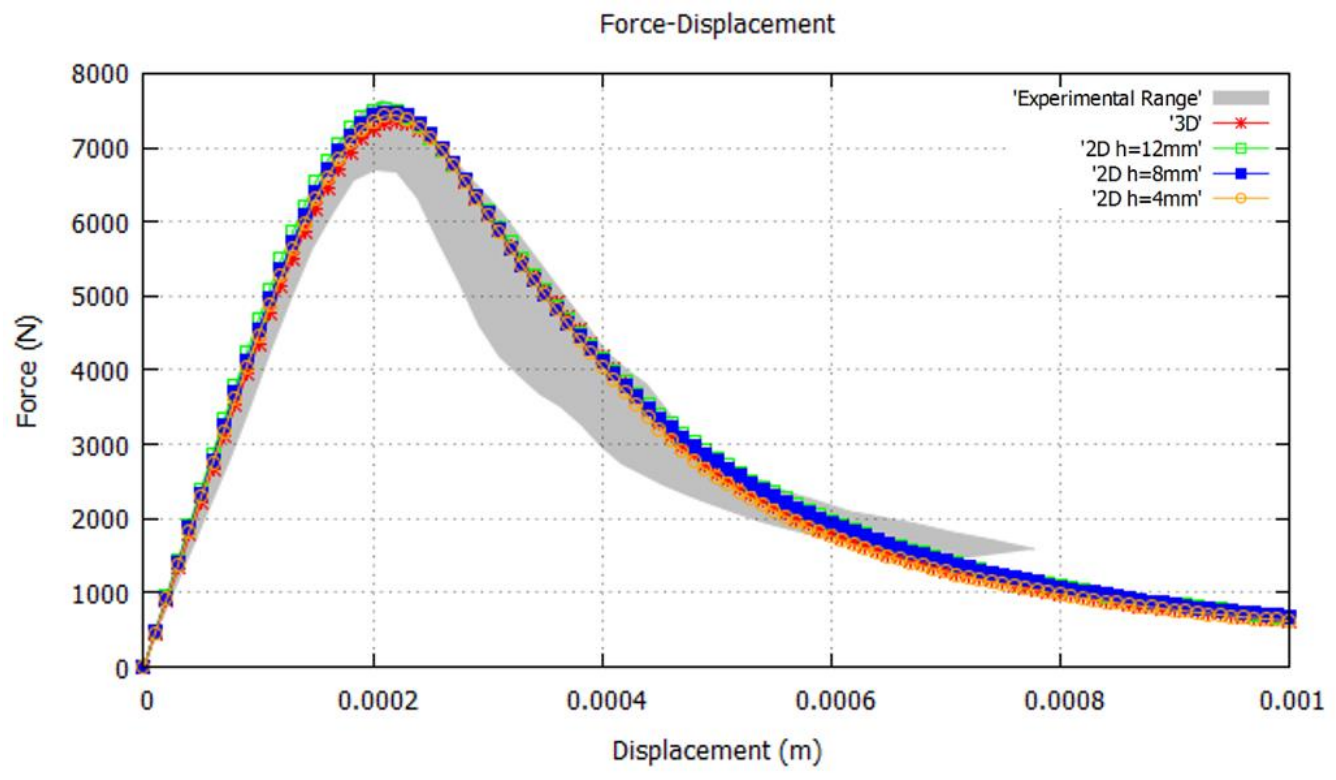

Figure 7. Force-displacement results for several mesh sizes

\section{Convergence of displacement and strain fields}

In order to illustrate the convergence of the displacement and strain fields provided by the smeared crack approach with mixed FE formulations, a vertical cut, shown in Figure 1 , is considered. Along this line, the profiles of vertical displacements and major principal strains are shown in Figure 8 for several load steps and in Figure 9 for different mesh sizes.

Figure 8, left column, shows how the displacement jump across the crack (CMOD) evolves as the load increases; it can be observed that, in all the meshes, the displacement jump is smeared across one element, an optimal representation of a strong discontinuity for the given mesh resolution. Figure 9, left, shows that the results practically overlap, demonstrating mesh size independence.

Figure 8, right column, shows the corresponding evolution of the strains as the load increases and the crack is formed. Note that: (a) the strain field is continuous, (b) the effective width of the strain localization band is $2 \mathrm{~h}$, (c) the value of the peak strain is inversely proportional to the mesh resolution, (d) the numerical solution approximates the Dirac's delta derivative of a discontinuous displacement field, as can be seen in Figure 9, right. 

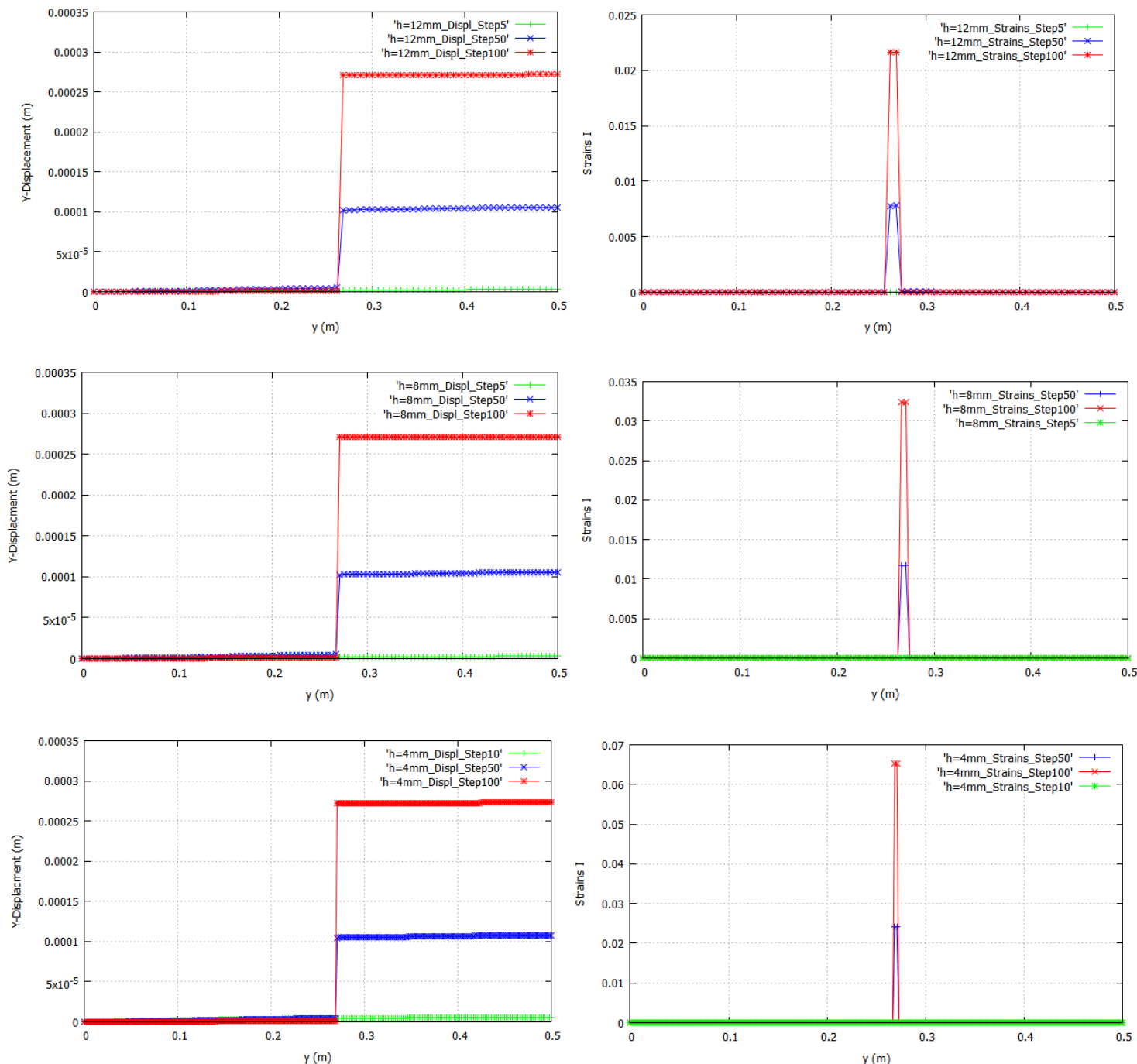

Figure 8. Vertical displacement and major principal strains along a vertical line for different load steps
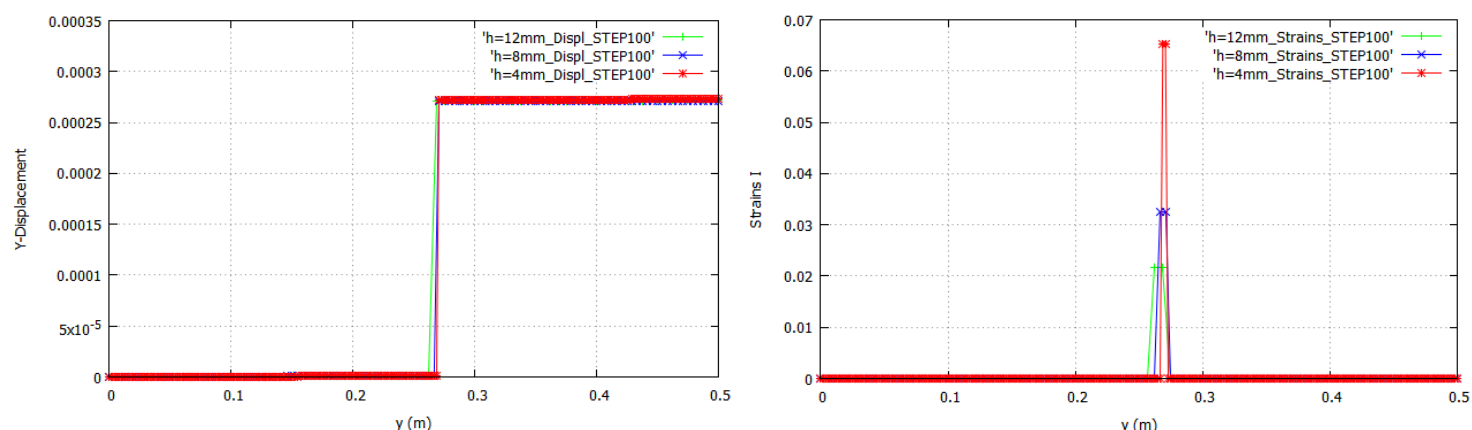

Figure 9. Vertical displacement and major principal strains along a vertical line for diferent sized meshes 


\subsection{Wedge-Splitting test}

In this second example, a wedge-splitting test of a concrete specimen is considered. The test was experimentally carried out by [69]. Reference [28] found similar results using embedded crack methods, as did reference [34] using XFEM. Reference [43] used embedded crack methods and crack tracking to compute a specimen with the same shape but in a reduced size.

The geometry is depicted in Figure 10 and the material properties are given in Table 2. The detail of the load application is shown in Figure 10. The thickness of the specimen is $0.4 \mathrm{~m}$.

This problem is solved using 2D triangular and 3D prism elements. The 2D computational domain is discretized with an unstructured mesh with elements of $40 \mathrm{~mm}$ of size, resulting in 7,488 nodes for 2D. The numerical analysis is carried out under the hypothesis of plane stress. The 3D mesh is obtained by the out-of-plane extrusion of the $2 \mathrm{D}$ mesh resulting in a semi-structured mesh of 29,952 nodes.

Table 2. Material parameters of the wedge-splitting test

\begin{tabular}{|c|c|}
\hline Young's Modulus & $28.3 \cdot 10^{9} \mathrm{~Pa}$ \\
\hline Poisson's Ratio & 0.2 \\
\hline Tensile Strength & $2.27 \cdot 10^{6} \mathrm{~Pa}$ \\
\hline Fracture Energy & $420 \mathrm{~J} / \mathrm{m}^{2}$ \\
\hline
\end{tabular}

This problem is solved using an arc-length algorithm controlling the crack mouth opening displacement (CMOD) at the points of load application.

Figure 11 shows the tensile damage distribution in the specimen for an imposed horizontal displacement of $5 \mathrm{~mm}$ obtained in the $2 \mathrm{D}$ and $3 \mathrm{D}$ analyses. Both results are identical because the same mesh configuration is used in the XY plane for the $2 \mathrm{D}$ and the $3 \mathrm{D}$ cases. These results agree with the experimental tests carried out by [69]. The crack path is vertical, as expected because of the symmetry of the geometry and the loading conditions. The crack surface obtained in the 3D analysis is shown in Figure 12, displayed as the level set surface of X-displacements. No auxiliary crack tracking technique has been used. No spurious mesh bias is observed.

Figure 13 shows the load vs. crack mouth opening displacement curve in the 2D and 3D cases, which are also compared to the results from the tests in [69]. Again, 2D and 3D results are almost overlapping. As can be seen, the results are very close to the experimental tests. They are also very close to the computational results of references [34], [28] and [43]. 


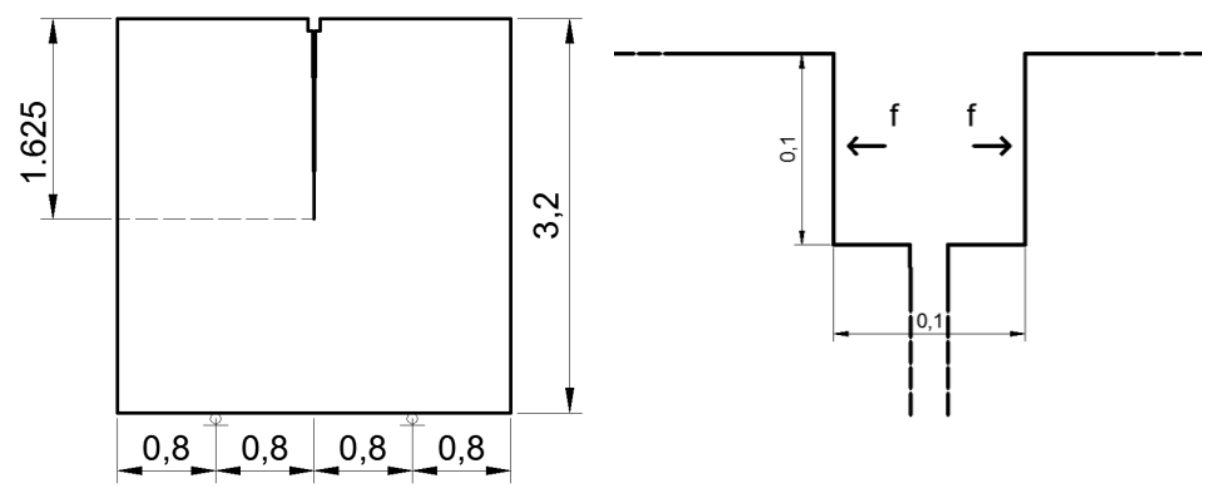

Figure 10. Geometry of the wedge-splitting test and detail of load application

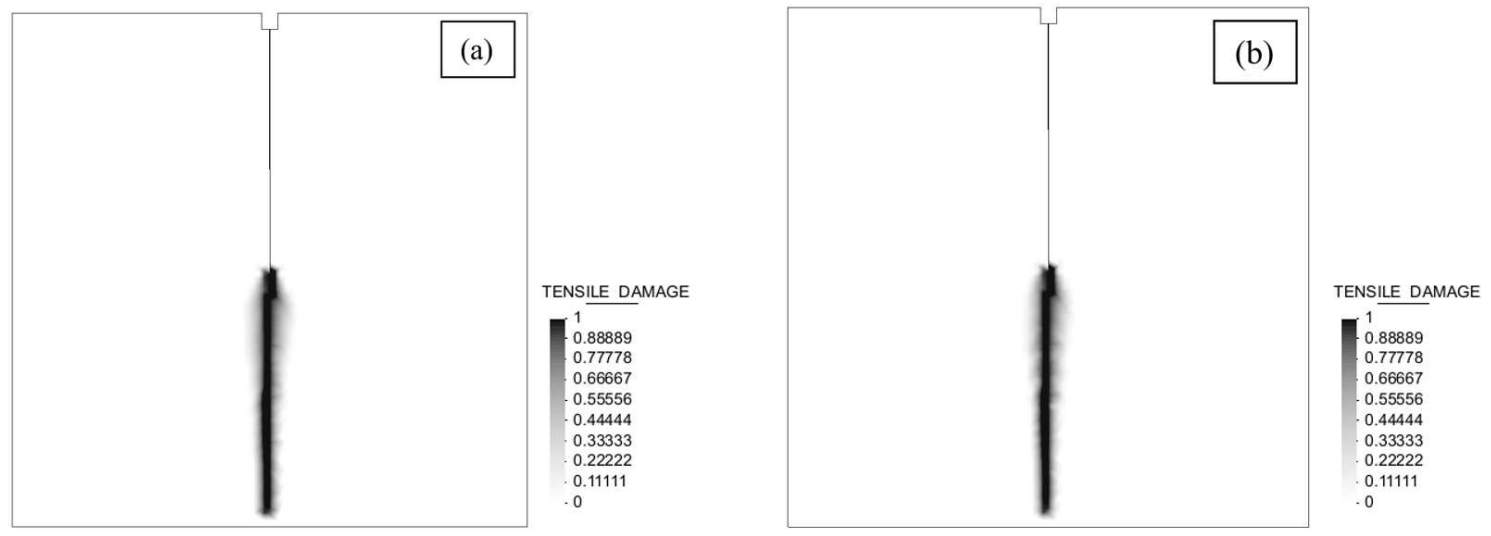

Figure 11. Tensile damage contour fills of the wedge-splitting test, (a) 2D and (b) 3D solution

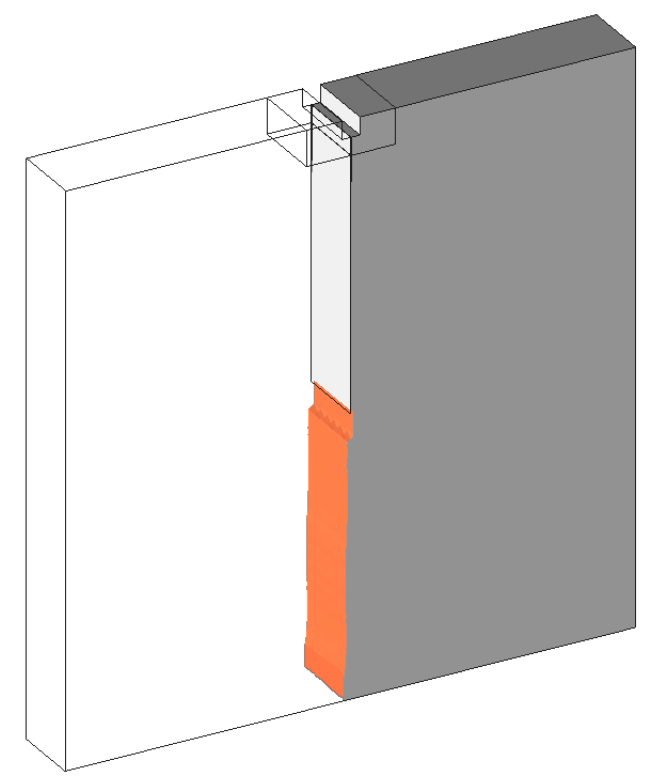

Figure 12. Crack surface of the modelled wedge-splitting test 


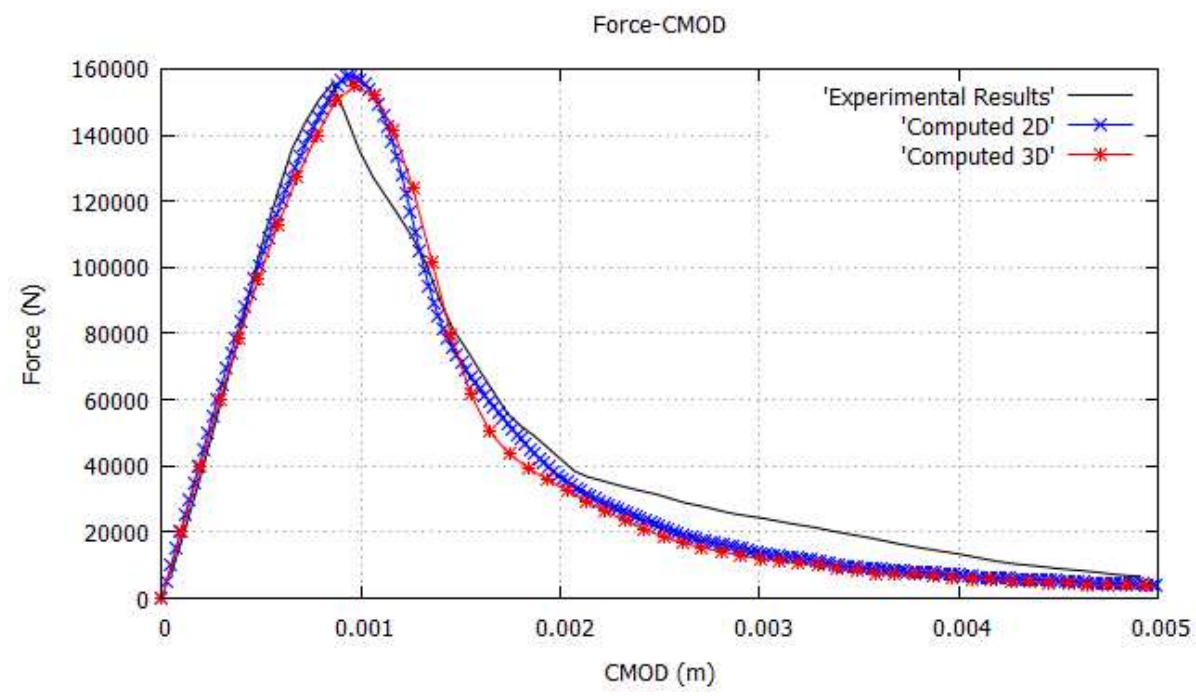

Figure 13. Force-CMOD results for $2 D$ and $3 D$ analysis.

\subsection{Three-point and four-point bending tests}

In this section, beams subjected to three-point and four-point bending tests are considered. The experimental tests were carried out by [70]. Other numerical results are reported in [71] and [34], where crack tracking auxiliary techniques are used. In Figure 14 the geometry of the tested beams is shown. Two cases are considered. In the first one, the stiffness at the upper left support is assumed equal to zero $(\mathrm{K}=0)$, as in a three-point bending test, while in the second one it is considered infinite $(\mathrm{K}=\infty)$, as in a four-point bending test. The thickness of the specimen is $0.05 \mathrm{~m}$. The properties of the material are given in Table 3. For the 2D analysis, plane stress conditions are assumed.

Table 3. Material properties of the beams

\begin{tabular}{|c|c|}
\hline Young's Modulus & $38.0 \cdot 10^{9} \mathrm{~Pa}$ \\
\hline Poisson's Ratio & 0.2 \\
\hline Tensile Strength & $3.0 \cdot 10^{6} \mathrm{~Pa}$ \\
\hline Fracture Energy & $69 \mathrm{~J} / \mathrm{m}^{2}$ \\
\hline
\end{tabular}

The problem is solved using an arc-length algorithm controlling the crack mouth opening displacement (CMOD) at the notch.

For this example, 2D triangular and 3D tetrahedral elements are used. In the 2D analysis, the computational domain is discretized with a fully unstructured mesh with $2.5 \mathrm{~mm}$ elements, resulting in 18,738 nodes. For the 3D analysis, a fully unstructured tetrahedral mesh of 50,389 nodes and $2.5 \mathrm{~mm}$ element-size has been considered. Both meshes are shown in Figure 15.

Figures 16 and 17 show plots of the computed tensile damage index in the cases considered. It can be observed that the crack path changes significantly depending on the boundary conditions applied to the beam. The $2 \mathrm{D}$ and $3 \mathrm{D}$ results for each case are almost 
overlapping. In Figure 18 the crack surfaces in the 3D analyses are shown, plotted as an iso-level surface of the X-displacements. There can be seen the fully unstructured mesh used for the computation of the beams, which is fine enough to model the crack surface with precision. As can be seen in Figure 19, the numerical results agree with the experimental tests. References [71] reported very similar crack paths using a global tracking algorithm. Also, [34] found very similar results using XFEM and the same global tracking algorithm.

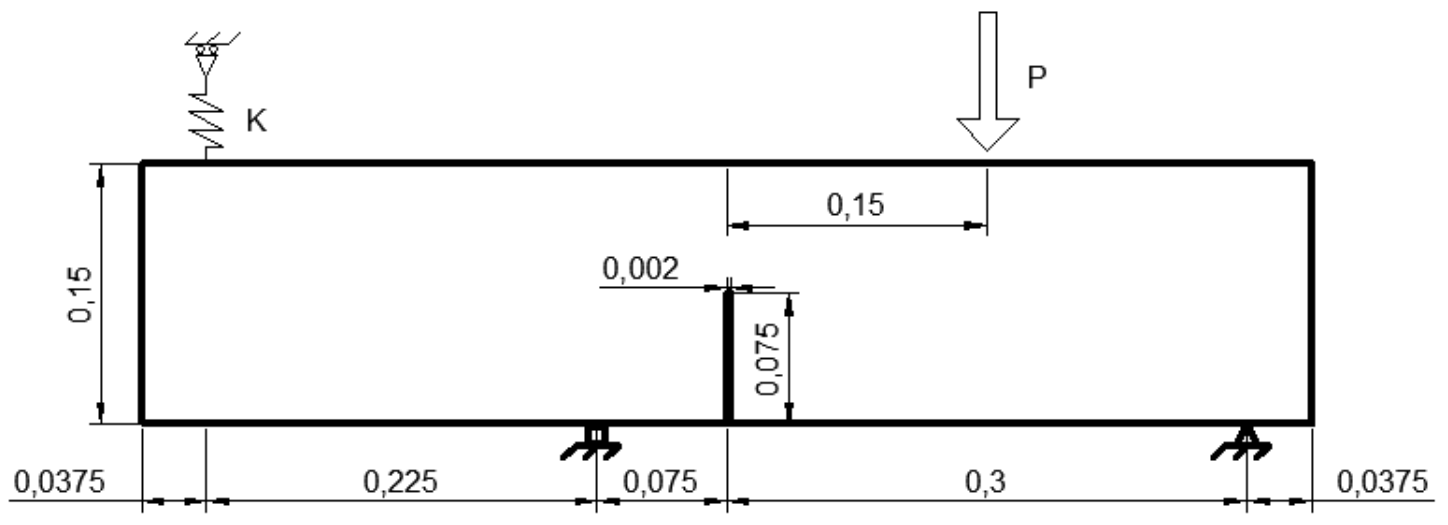

Figure 14. Geometry of the three-point and four-point beam (m)
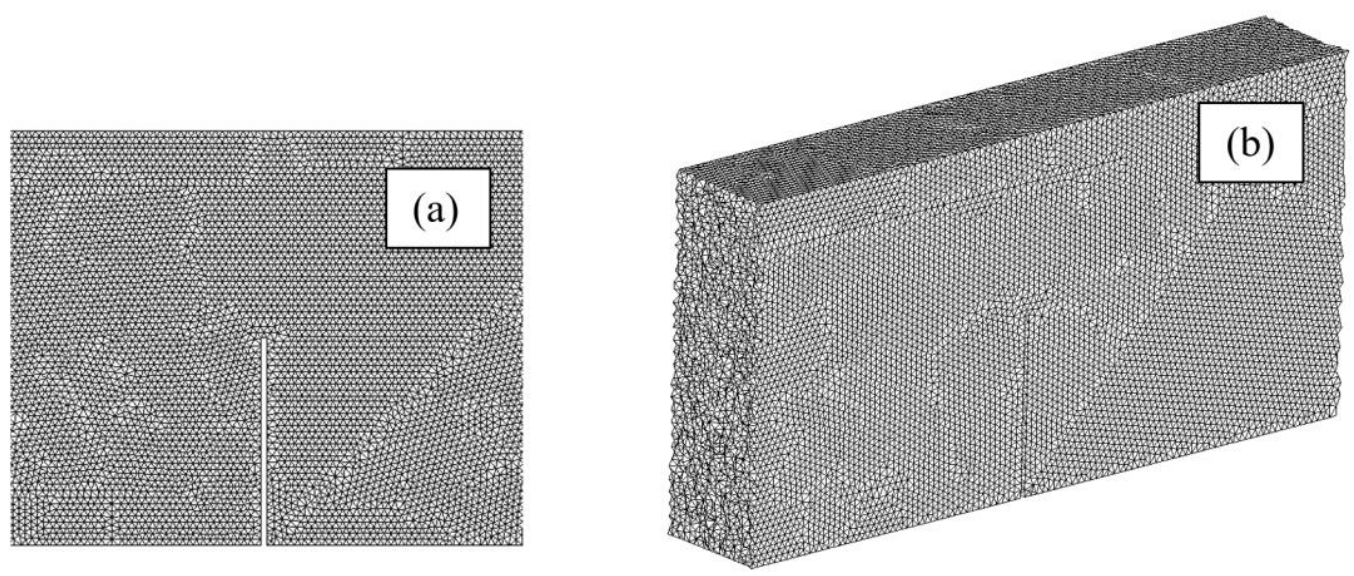

Figure 15. Meshes used for the (a) 2D and (b) 3D analyses of the three-point and the four-point bending tests
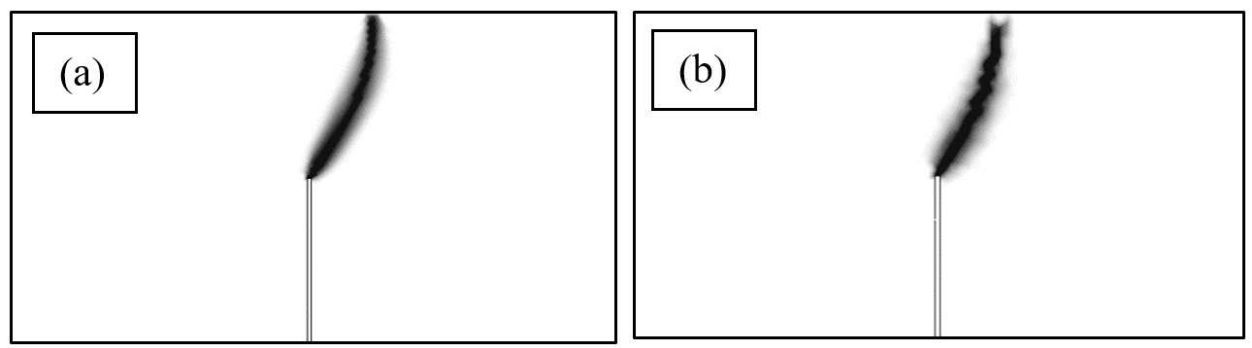

Figure 16. Tensile damage contour fills of the three-point bending test, (a) 2D solution, (b) 3D solution 

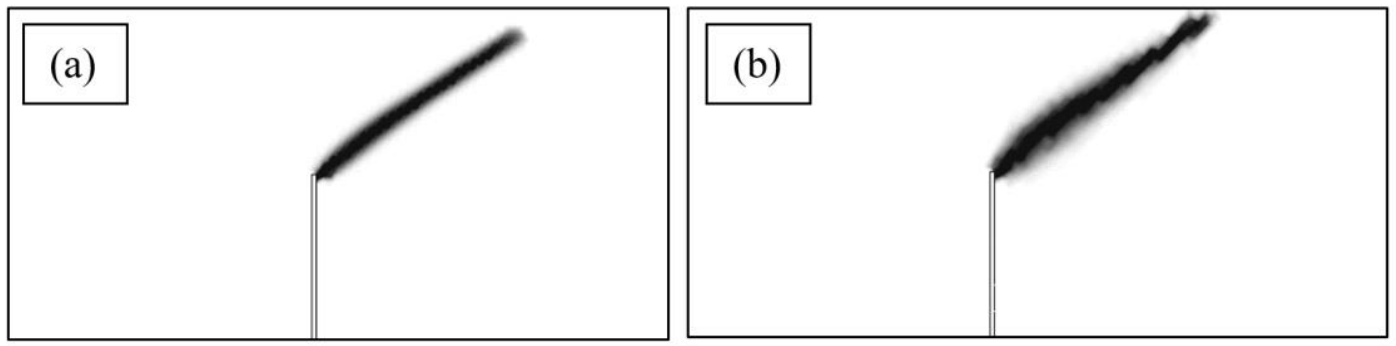

Figure 17. Tensile damage contour fill of the four-point bending test, (a) 2D solution, (b) 3D solution
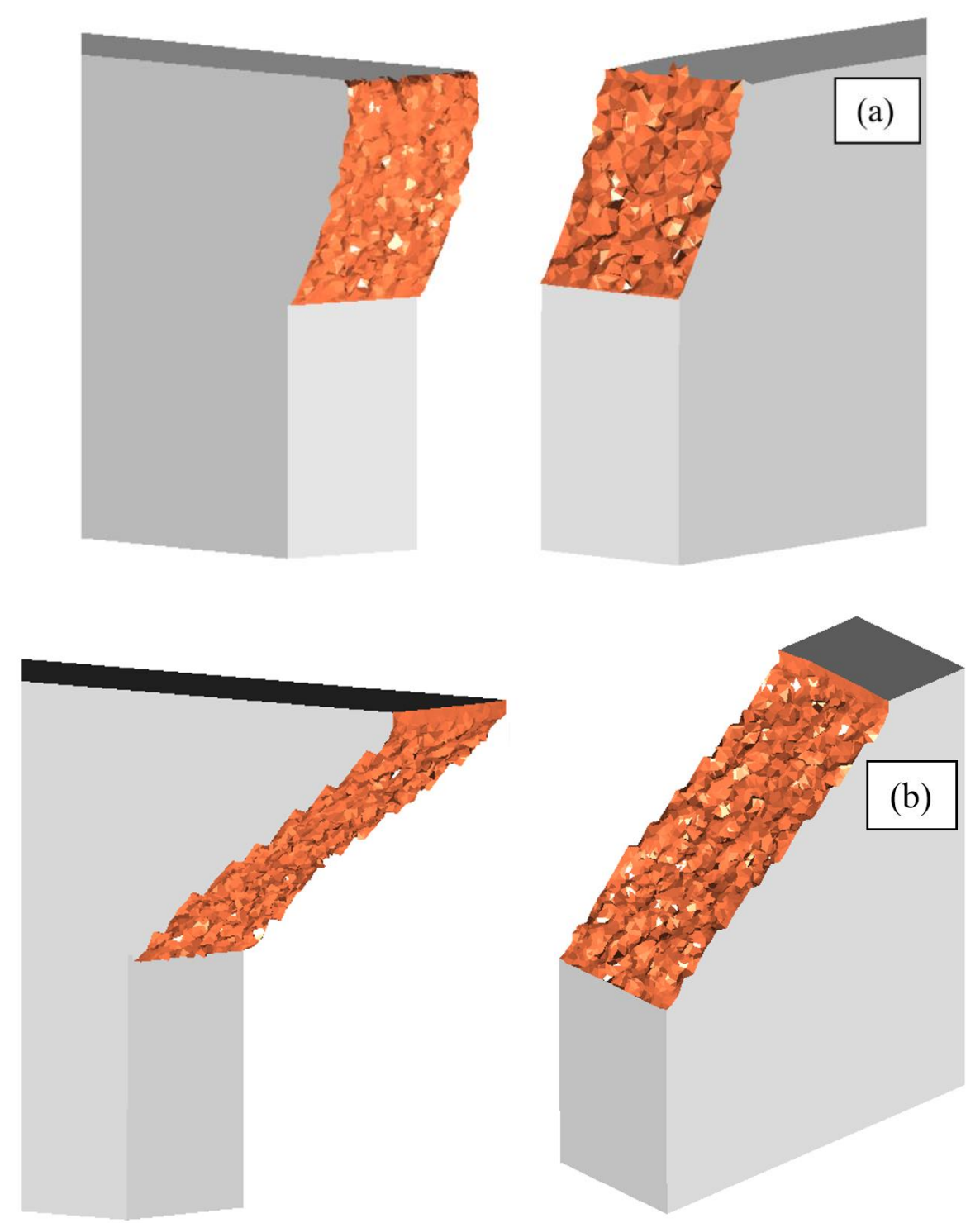

Figure 18. Modelled crack surfaces for the (a) three-point and (b) four-point bending cases 

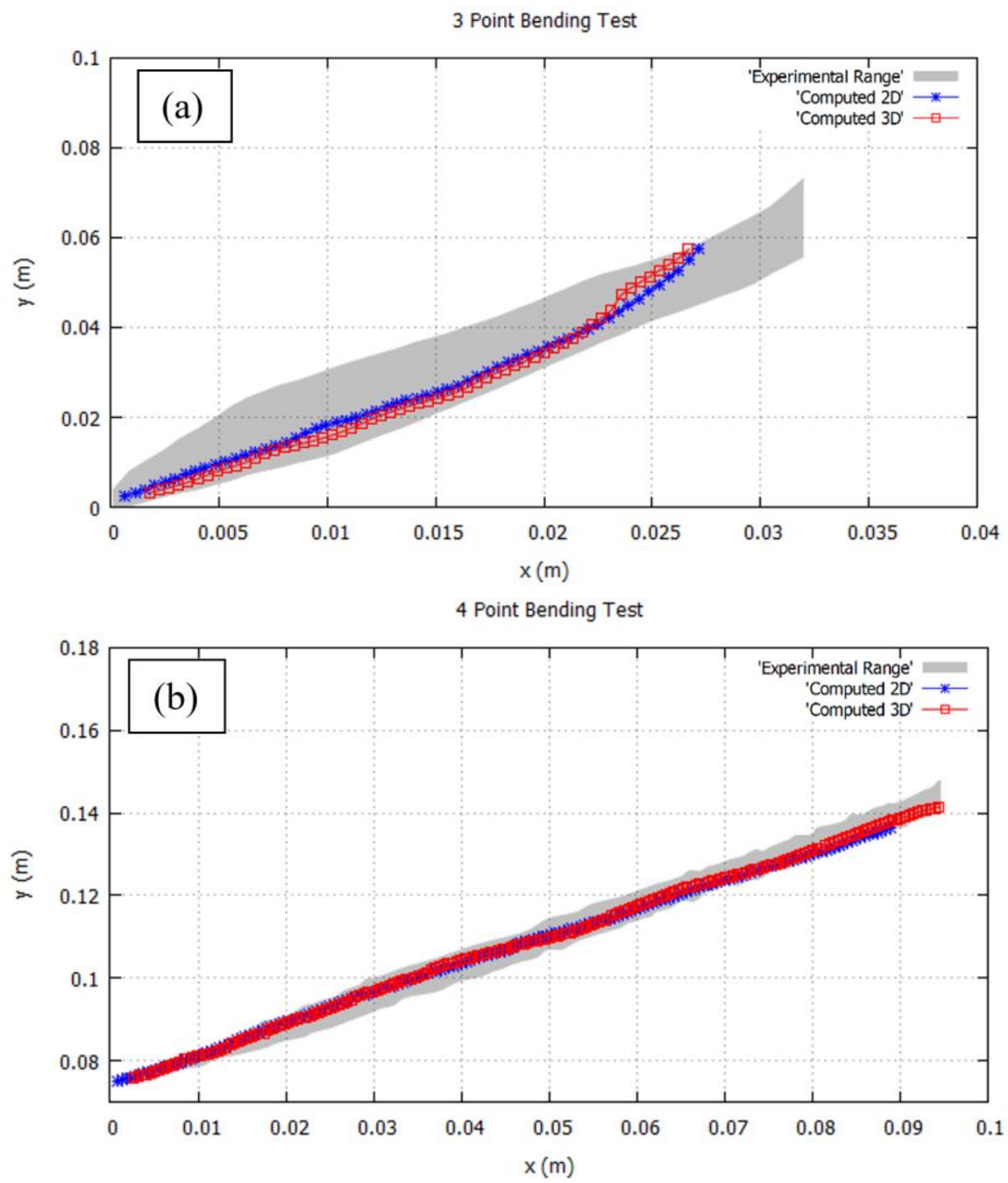

Figure 19. Computed crack paths compared to experimental results for (a) three-point and (b) four-point bending cases

Figure 20 shows the load-CMOD curves for both the 3 and 4 point bending tests. The results are similar to the ones obtained in references [71] and [34]. The three-point bending test shows very good agreement with the experimental results obtained by [70] both in the 2D and 3D analyses, even if at the last stages of the simulation the strength is slightly underestimated. The peak force is slightly lower in the 3D analysis. The fourpoint test has its peak slightly outside the experimental range of results. This occurs also in the numerical references [71] and [34]. 

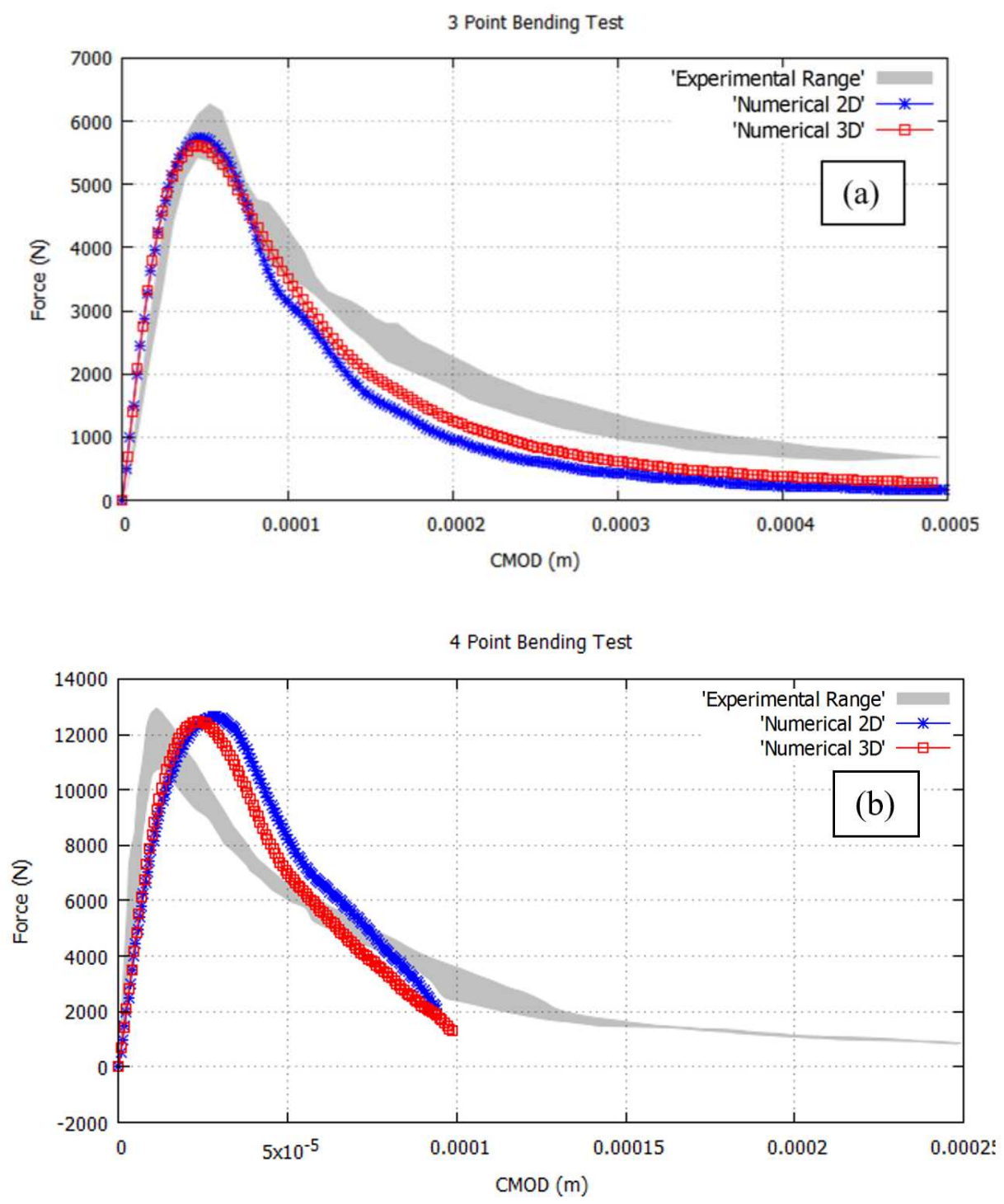

Figure 20. Load-CMOD curves for (a) the three-point and (b) four-point bending tests

\section{Structural size effect}

Structural size effect addresses the question of how the load capacity of geometrically similar structures varies when scaling up or down their relative sizes. Experimental evidence shows that, for a given structural problem, ductile behavior corresponds to the small scale limit (appropriate for small laboratory specimens), while brittle fracture occurs in the large scale limit (apt for structures of very large dimensions). Thus, it is of practical interest to develop analytical and numerical tools suitable to bridge the gap between perfectly ductile and perfectly brittle behavior, i.e. suitable for the range of quasibrittle failure [54].

In quasi-brittle fracture, size effect does not only affect the load capacity (peak load), it also reflects on the post-peak behavior (ductility) of the structure. The capability of a 
quasi-brittle structure to absorb energy decreases, in relative terms, as the structure size increases [54].

In reference [70], structural size effect was investigated when testing the beams. For this, smaller specimens with half the original size, i.e. a height of $\mathrm{D}=75 \mathrm{~mm}$, and double size, i.e. $\mathrm{D}=300 \mathrm{~mm}$ of height, where also experimentally tested. These cases have also been simulated computationally for the three-point beam and are reported in the following.

In Figure 21, the Force-CMOD curves of the three considered cases, small ( $D=75 \mathrm{~mm}$ ), medium $(D=150 \mathrm{~mm})$ and large $(D=300 \mathrm{~mm})$, are shown. The computed results show very good agreement with the experimental ones of reference [70], even if the dissipated energy is slightly underestimated. In Figure 22, the computed crack paths also show very good agreement with the experimental results for the small and large specimens.

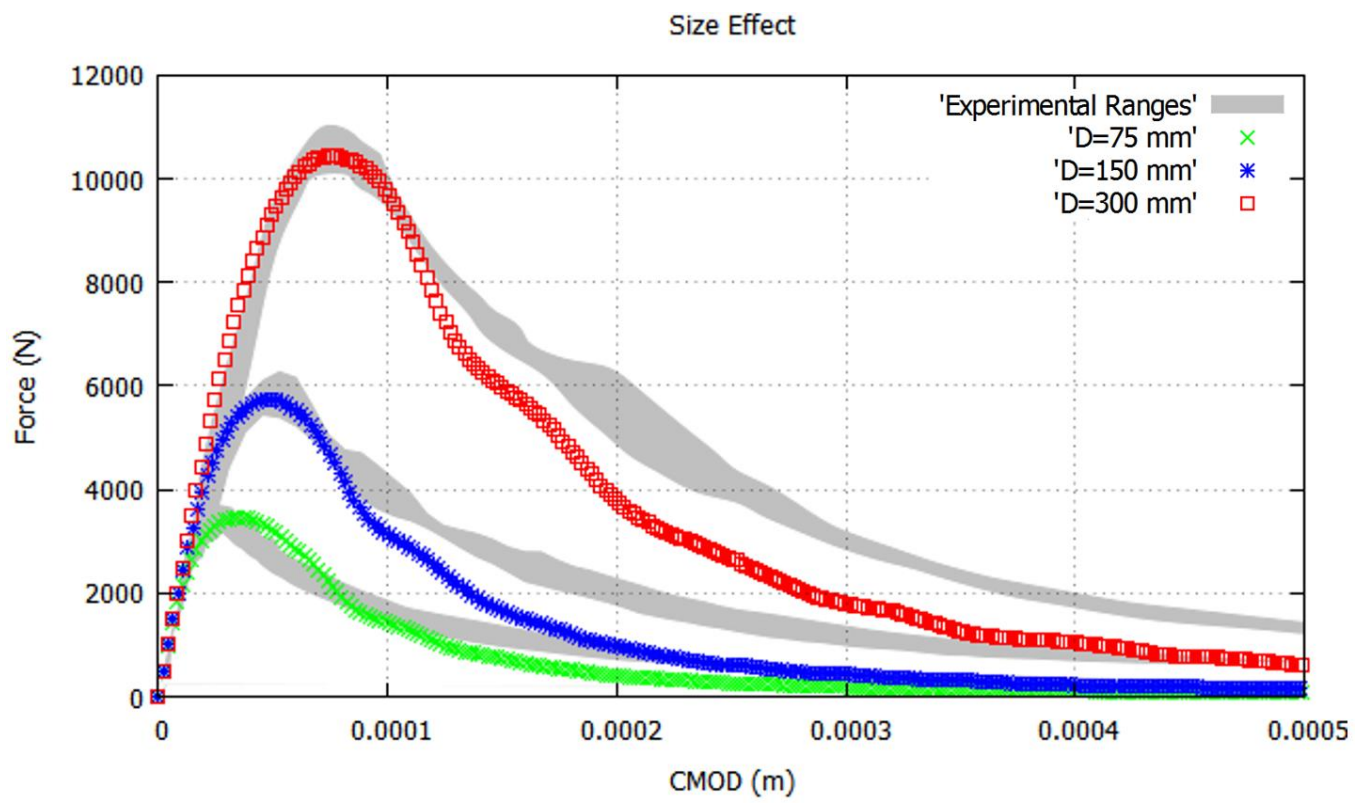

Figure 21. Force-CMOD curves for different beam sizes 

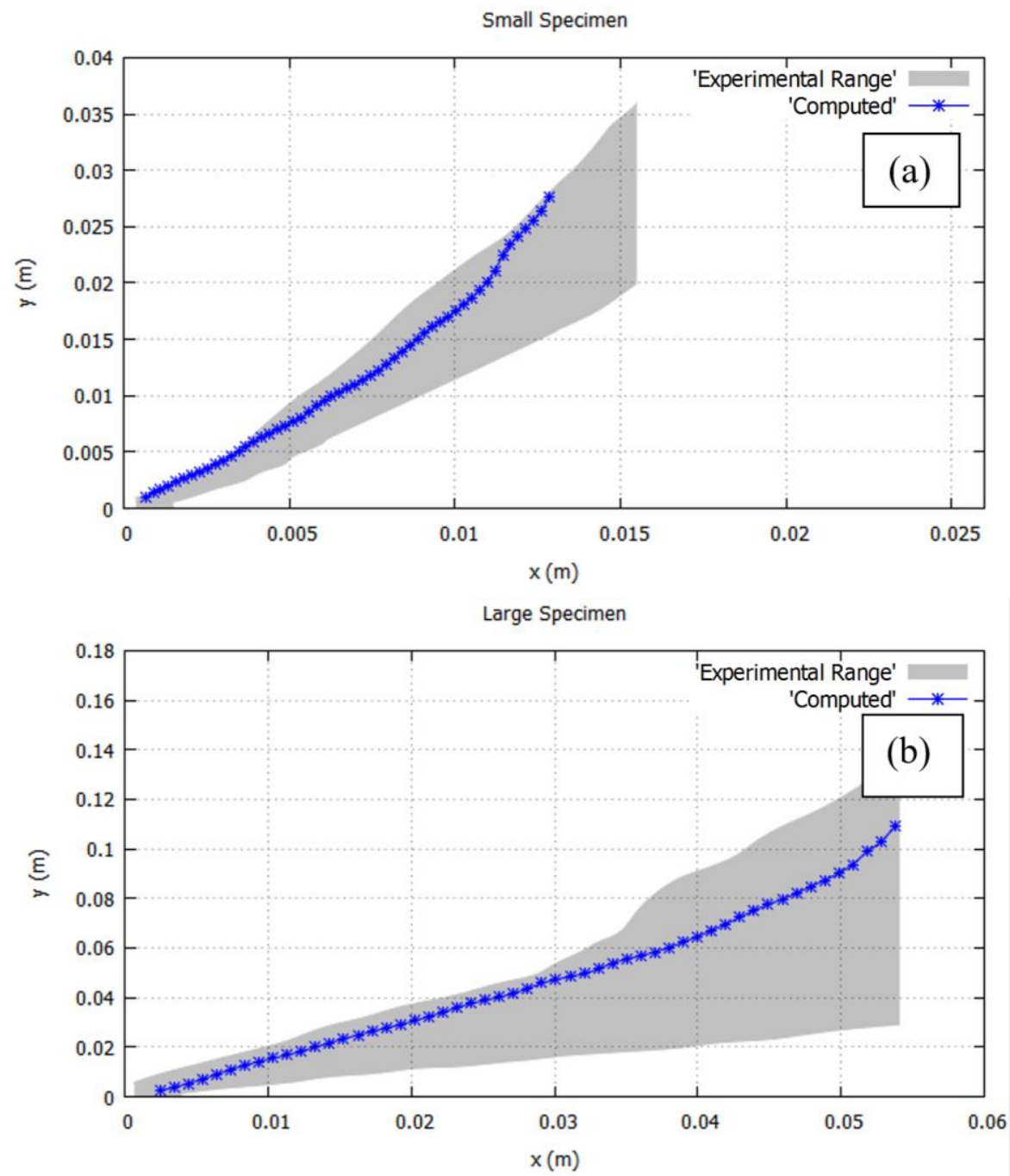

Figure 22. Crack path in (a) the small specimen and (b) the large specimen

\section{Plane Strain vs Plane Stress}

The plane stress hypothesis adopted in the 2D computation may be verified by comparing the results obtained against those obtained under plane strain assumptions. Figure 23 shows the force-CMOD results for both considerations. The 3D analysis is also included. All three cases are almost overlapping and equally close to the experiments. This indicates that the standard engineering practice of neglecting Poisson's effect in beam theory can be extended into the non-linear range. 
Plane Strain vs Plane Stress

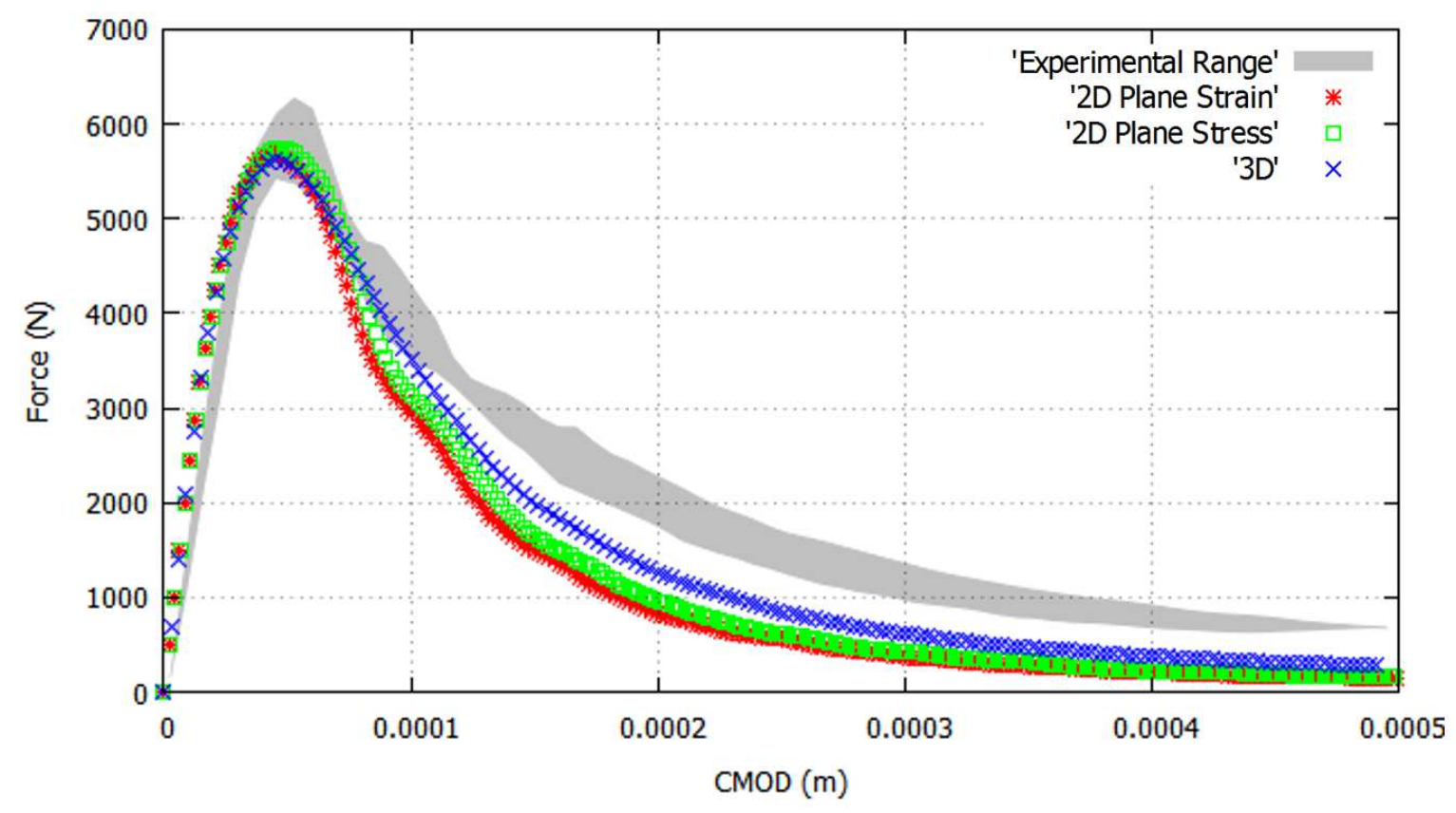

Figure 23. Comparison of 2D plane strain, 2D plane stress and 3D hypothesis

\subsection{Notched beams with holes}

A more involved example is considered in this section to explore the performance of the proposed finite element formulation. Here, a notched beam with holes, experimentally tested and numerically computed by [72], is considered. Other numerical solutions are reported in [41], [72] and [73]. Reference [41] modelled this example using crack tracking and embedded methods and [73] used fracture mechanics. Instead [72] used a probabilistic approach, where geometric and material uncertainties are considered when computing the crack path and load-displacement curves.

The tested beam is made of plexiglass; the properties used for the simulation are given in Table 4.

For comparison purposes, three different geometries regarding the position of the notch and the holes, shown in Figure 24, are studied. In the original experiment inches were used as units of length.

In the first case, the beam is notched but has no holes; the notch is 6" from the center and 1 " long. In the second case, the beam has three holes of diameter 0.5 " at 4 " from the center and a notch identical to the previous case. In the third case, the hole layout is the same as in the second case and the notch is 4.5 " from the center and is 1.5 " long. The thickness of the beam is $0.5^{\prime}$ '. For the $2 \mathrm{D}$ analysis, plane stress conditions are assumed. 

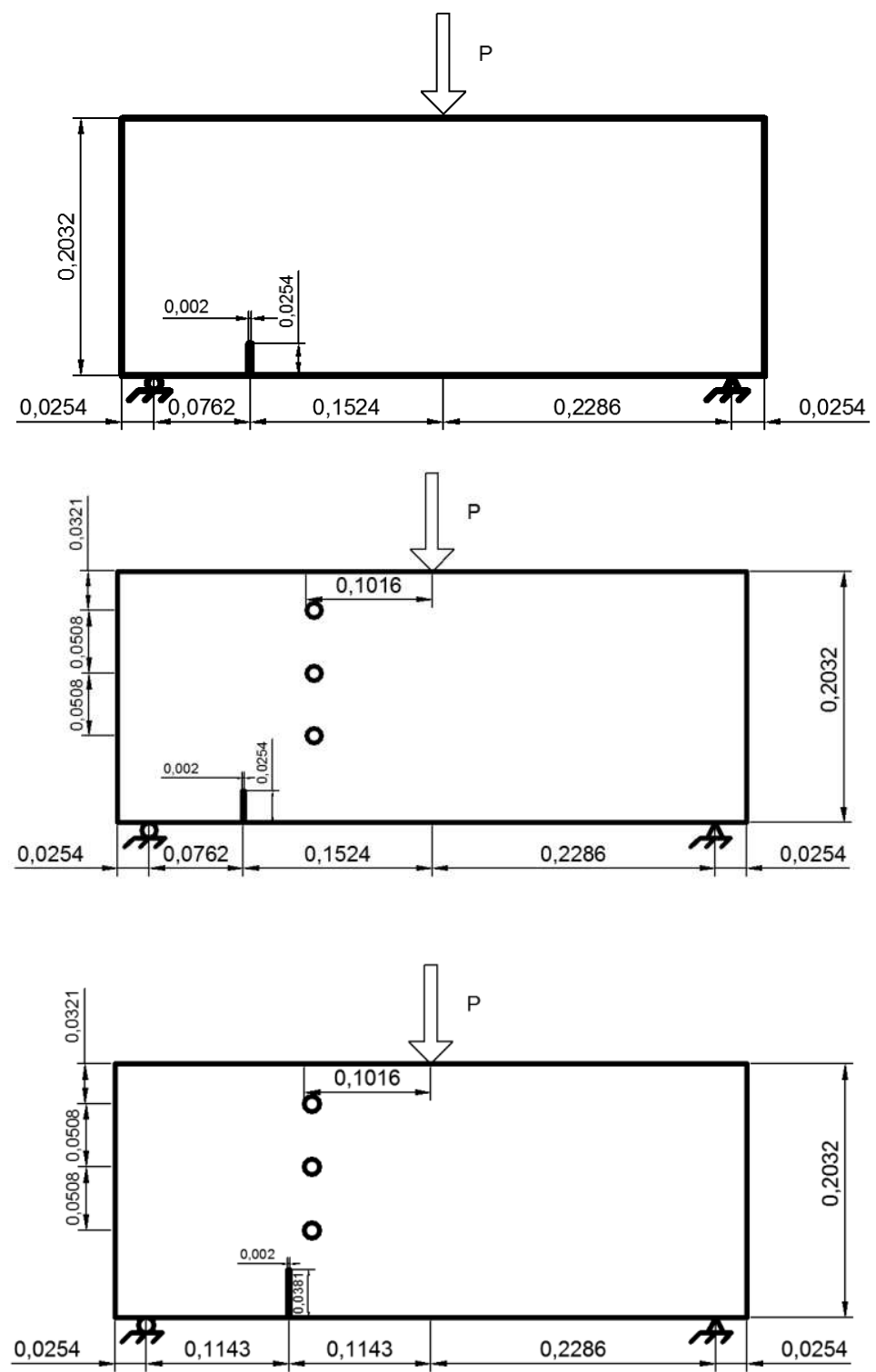

Figure 24. Geometry for the three notched beams (m)

Table 4. Material properties of the three holed notched beams

\begin{tabular}{|c|c|}
\hline Young's Modulus & $3.102 \cdot 10^{9} \mathrm{~Pa}$ \\
\hline Poisson's Ratio & 0.35 \\
\hline Tensile Strength & $7.0 \cdot 10^{6} \mathrm{~Pa}$ \\
\hline Fracture Energy & $500 \mathrm{~J} / \mathrm{m}^{2}$ \\
\hline
\end{tabular}




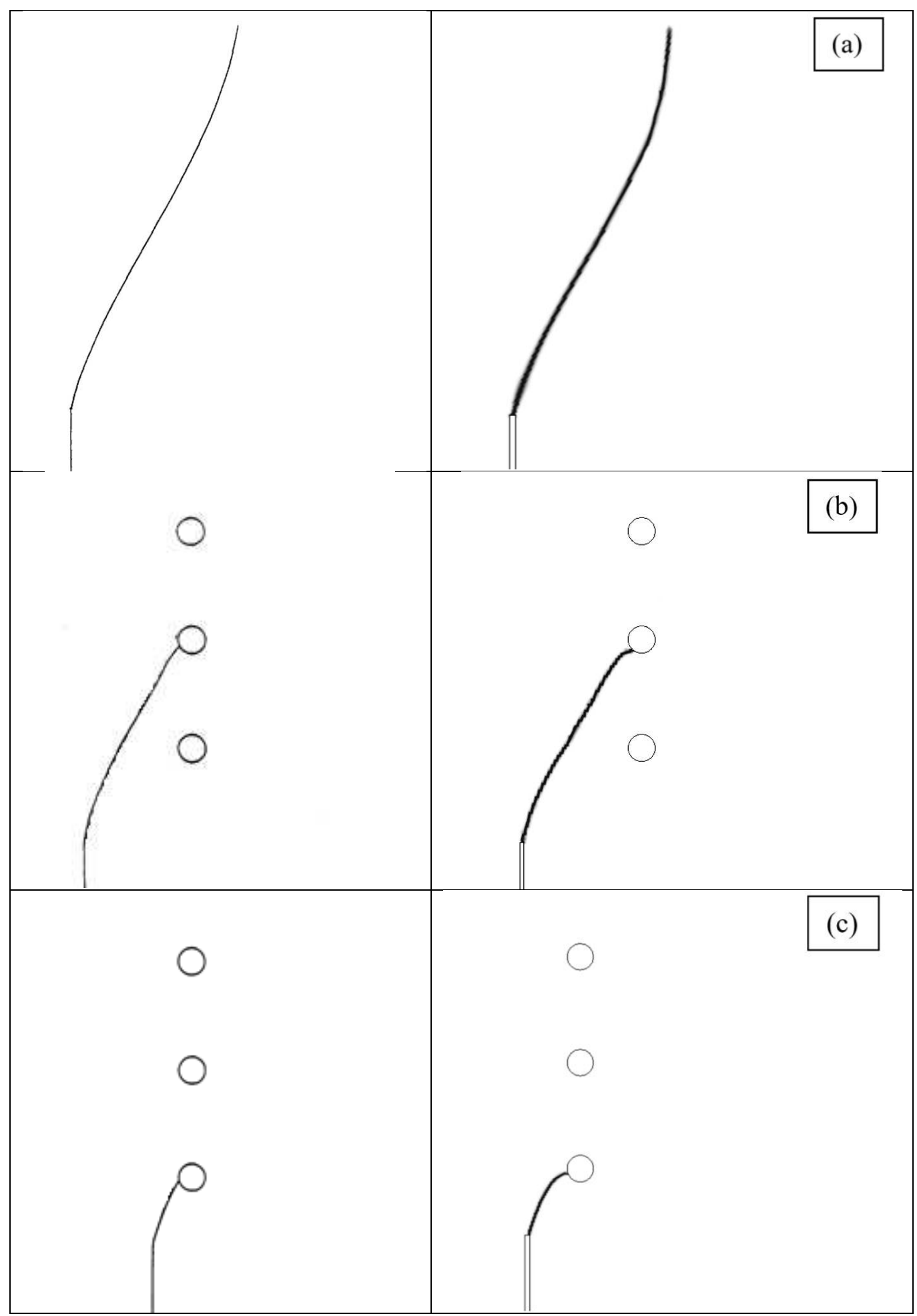

Figure 25. Experimental and computed crack paths for the three notched beams. Experimental results taken from [72]

In all cases, 2D triangular elements are used. The domain is discretized with unstructured meshes of $1 \mathrm{~mm}$ elements in the central part, where the crack forms, and of $2.54 \mathrm{~mm}$ in the rest of the beams, resulting in 44,956, 52,884 and 52,600 nodes, respectively.

The simulation is done using an arc-length algorithm controlling the crack mouth opening displacement (CMOD). 
Figure 25 shows the computed crack paths next to the experimental results reported in [72]. It can be observed that the crack paths are different depending on the notch position and the presence of the holes. The crack path for the case without holes is almost identical to the experimental result. In the other two cases, the numerical results also show good agreement with tests. The present results are comparable to those obtained in [41], [72] and [73].

Figure 26 shows the load-CMOD curve for the case without holes, the only one reported in [72]. The computed results show good agreement with the ones obtained experimentally. In Figure 27 the force vs displacement curve at the point of load application is presented. The numerical results are stiffer than the experimental ones. This was also observed in the numerical results reported by [72]. Note that the local response at the point of load application is very dependent on the actual details of the experimental set-up. Nevertheless, the overall resemblance of the results is remarkable, in terms of peak values, snap-back response and dissipated energy.

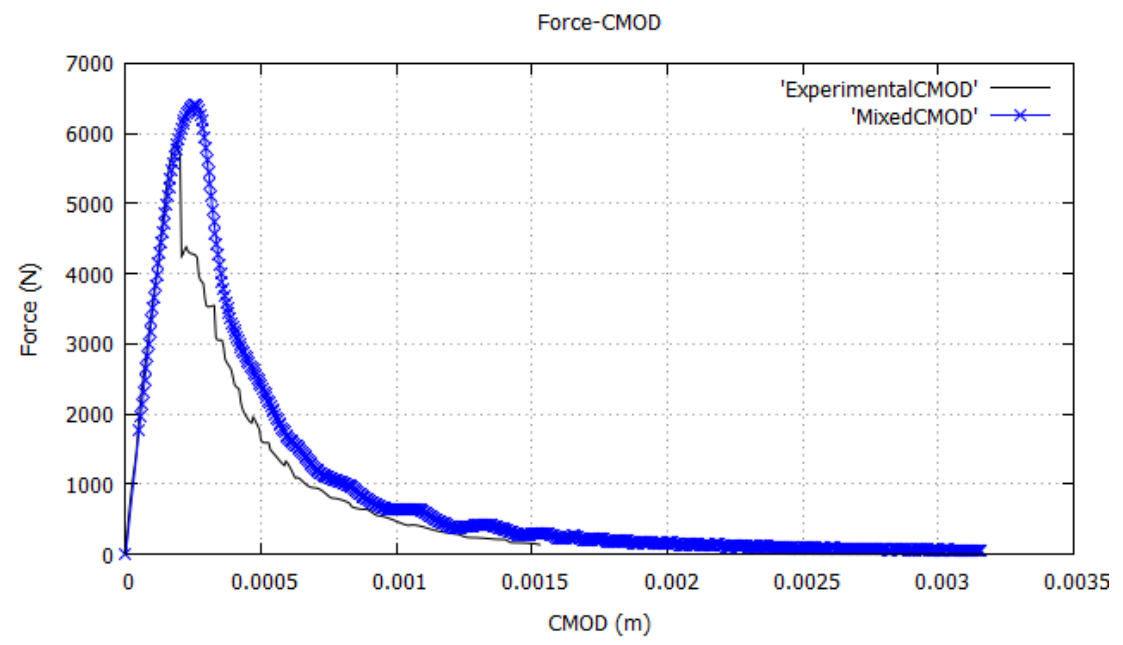

Figure 26. Force-CMOD curve for the notched beam without holes

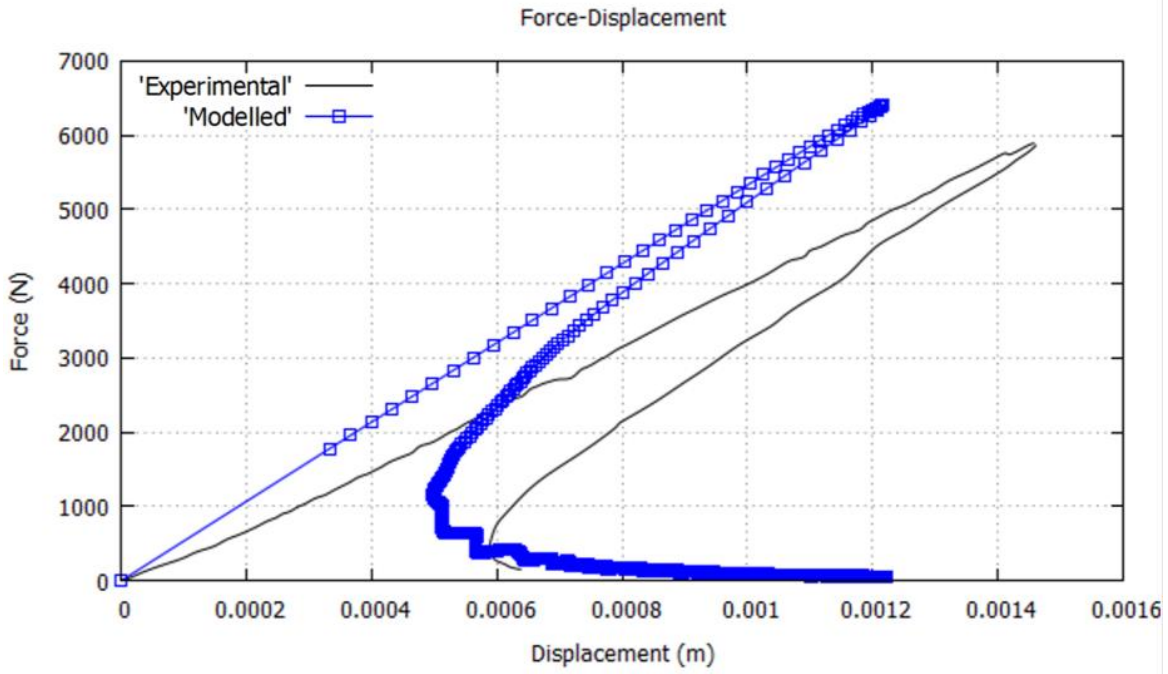

Figure 27. Force-displacement curve for the notched beam without holes 

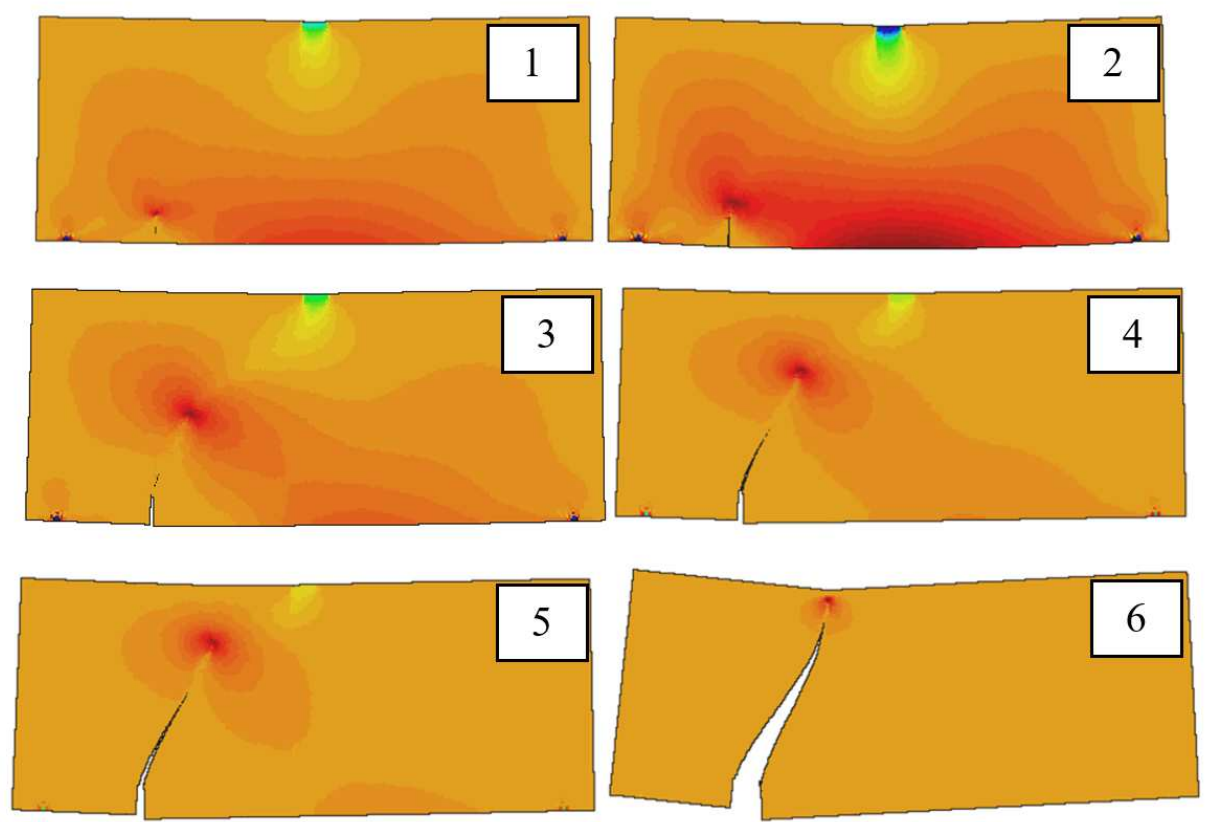

Figure 28. Crack propagation and evolution of major principal stresses in the notched beam without holes

Figure 28 shows the crack propagation and evolution of major principal stresses in the notched beam without holes. In the elastic range, stresses concentrate in the vicinity of the tip of the notch. This causes the crack to start and propagate through the height of the beam and towards the point of application of the load as also shown in Figure 25(a). The strain/displacement FE formulation is able to represent this progressive failure of the beam with noteworthy accuracy.

\subsection{Four-point bending test on a doubly-notched beam}

The numerical analysis of a four-point bending test on a doubly notched beam is considered next. The corresponding experiments are reported in [74]. Other numerical solutions are reported in [37, 75, 76]. In [37] an adaptive particle meshless method was used, while in [75] the boundary element method was employed. Reference [76] considers a localization limiter introduced to regularize the problem. All these simulations are in 2D.

The beam geometry and loading is shown in Figure 29 and the material parameters are given in Table 5. The thickness of the beam is $0.1 \mathrm{~m}$. The structural problem presents polar-symmetry about the geometrical center. Thus, two symmetric cracks are expected to appear, starting from the top of the notches and propagating towards the opposite top and bottom faces of the beam. The cracks open and propagate under mixed Modes I and II (opening and shearing) loading. The ratio between the uniaxial compressive and tensile strengths is 15 [74]. 
In this section, the problem is analyzed in $2 \mathrm{D}$, assuming plane stress conditions, and in $3 \mathrm{D}$. The objective is to assess the performance of the proposed formulation when more than one crack appears.

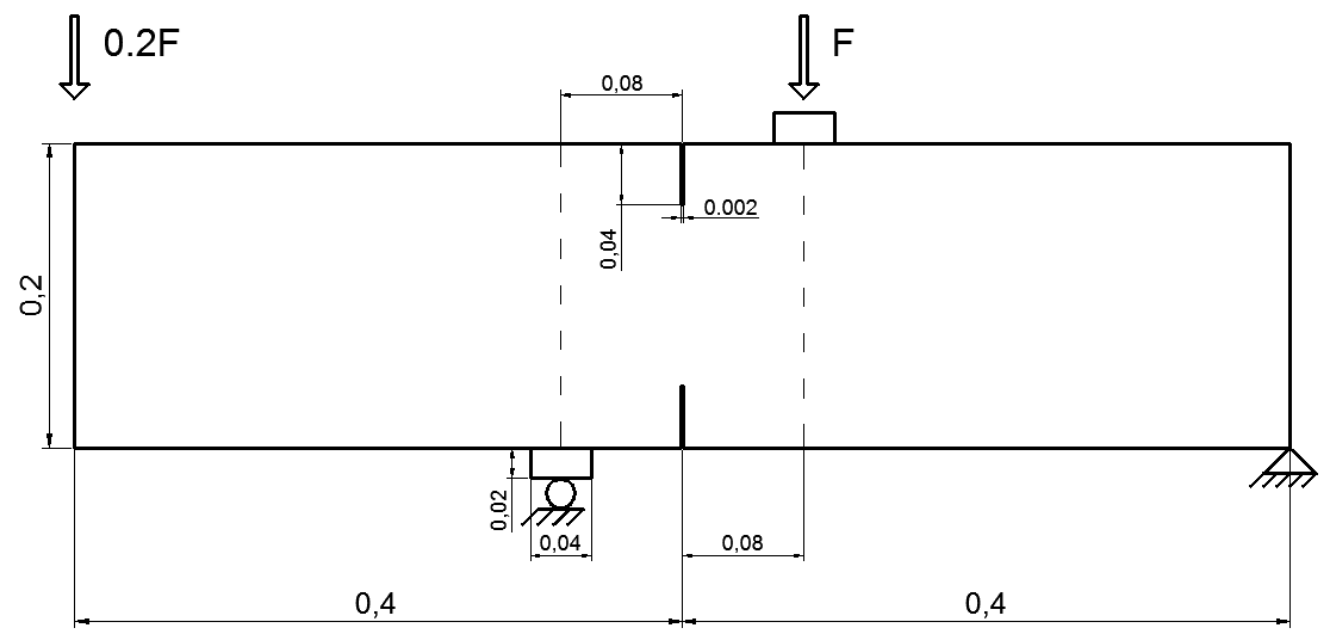

Figure 29. Geometry of the four-point bending test on a doubly-notched beam

Table 5. Material parameters of the four-point bending test on a doubly-notched beam

\begin{tabular}{|c|c|}
\hline Young's Modulus & $27 \cdot 10^{9} \mathrm{~Pa}$ \\
\hline Poisson's Ratio & 0.18 \\
\hline Tensile Strength & $2.0 \cdot 10^{6} \mathrm{~Pa}$ \\
\hline Fracture Energy & $100 \mathrm{~J} / \mathrm{m}^{2}$ \\
\hline Compressive Strength & $3.0 \cdot 10^{7} \mathrm{~Pa}$ \\
\hline
\end{tabular}
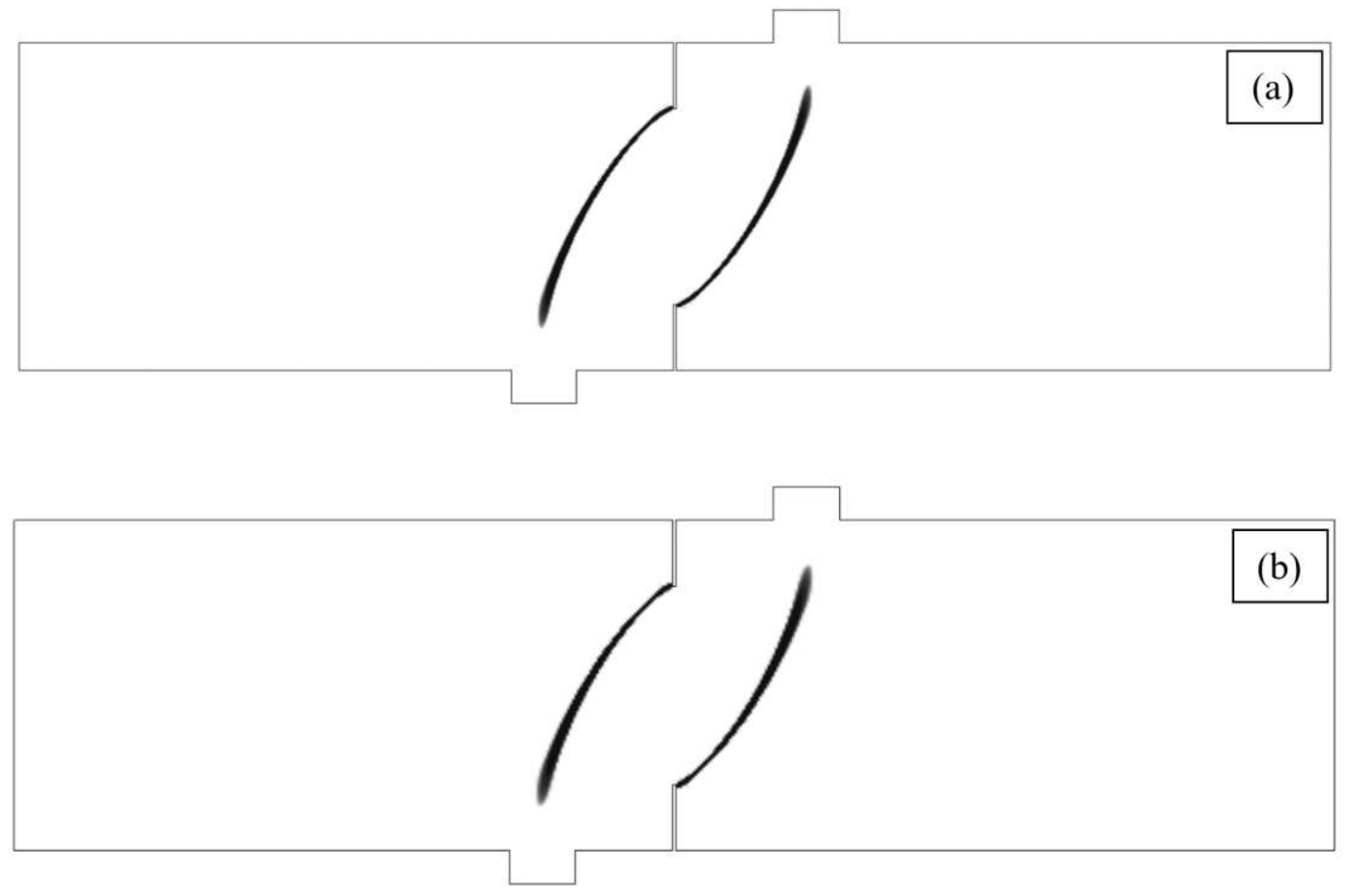

Figure 30. Tensile damage contour fills of the four-point bending test on a doubly notched beam for (a) the 2D and (b) the 3D computations 
The simulation is performed using an arc-length algorithm controlling the crack mouth opening displacement (CMOD) at the upper notch.

This example is solved using 2D quadrilateral elements and 3D hexahedra. In 2D the computational domain is discretized with a fully structured mesh of $1 \mathrm{~mm}$ size, resulting in a 54,021 node mesh. In 3D, the computational domain is partitioned using hexahedra elements of $1.5 \mathrm{~mm}$ size, ensuing a structured mesh of 53,998 nodes.

Figure 30 shows the tensile damage contour fills obtained in the 2D and 3D simulations. Due to the polar symmetry of the beam geometry and loading, two polar-symmetric cracks appear and propagate. These results are concordant with the ones observed in the experiments by [74]; the cracks propagate as expected. The computed 2D and 3D paths of both cracks are almost overlapping and are inside the experimental ranges obtained in the tests, as can be observed in Figure 31.

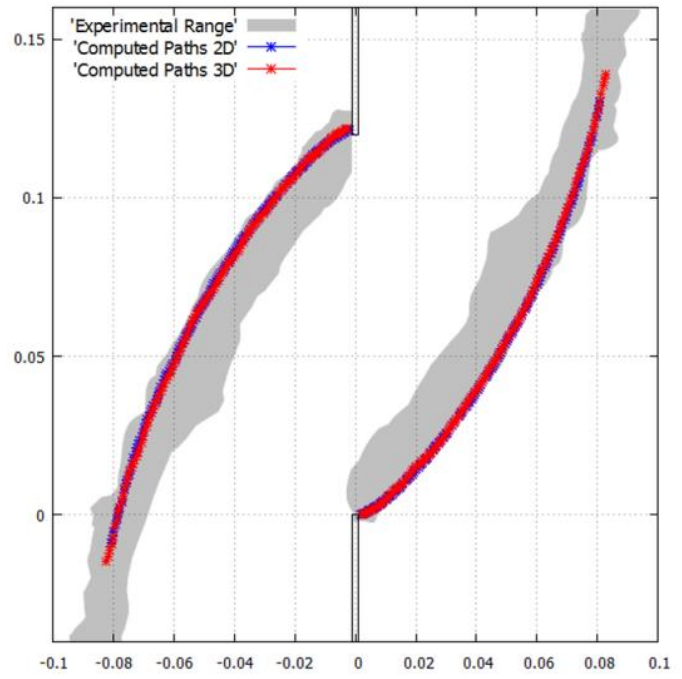

Figure 31. Crack path of the four-point bending test on a doubly notched beam

Also because of the polar symmetry of the problem, the central part of the beam rotates, with respect to the central point, as can be seen in Figure 32, where contour fills of the displacements are shown. The displacement "jump" across the cracks can also be observed neatly.

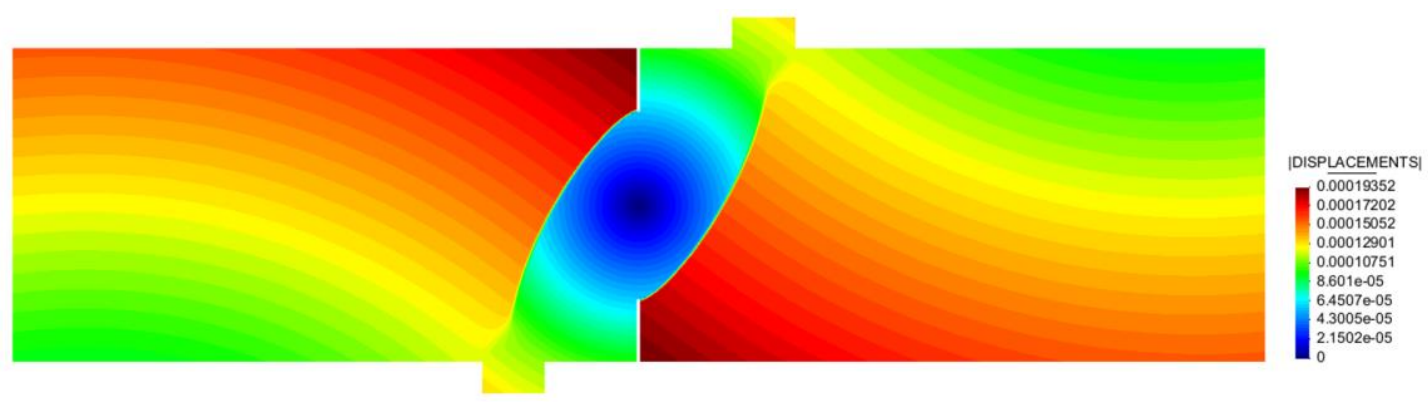

Figure 32. Displacements of the four-point bending test on a doubly notched beam

The crack surfaces in the 3D analysis are depicted in Figure 33, plotted as iso-level surfaces of the norm of displacements. In this way, the performed simulation allows to observe a $3 \mathrm{D}$ representation of the piece formed during the experiment. This case 
illustrates the performance of the proposed method to deal with several cracks propagating at the same time in $2 \mathrm{D}$ and $3 \mathrm{D}$. As no auxiliary tracking technique is required, the present formulation can handle this situation, which does not represent an extra hindrance to the formulation.
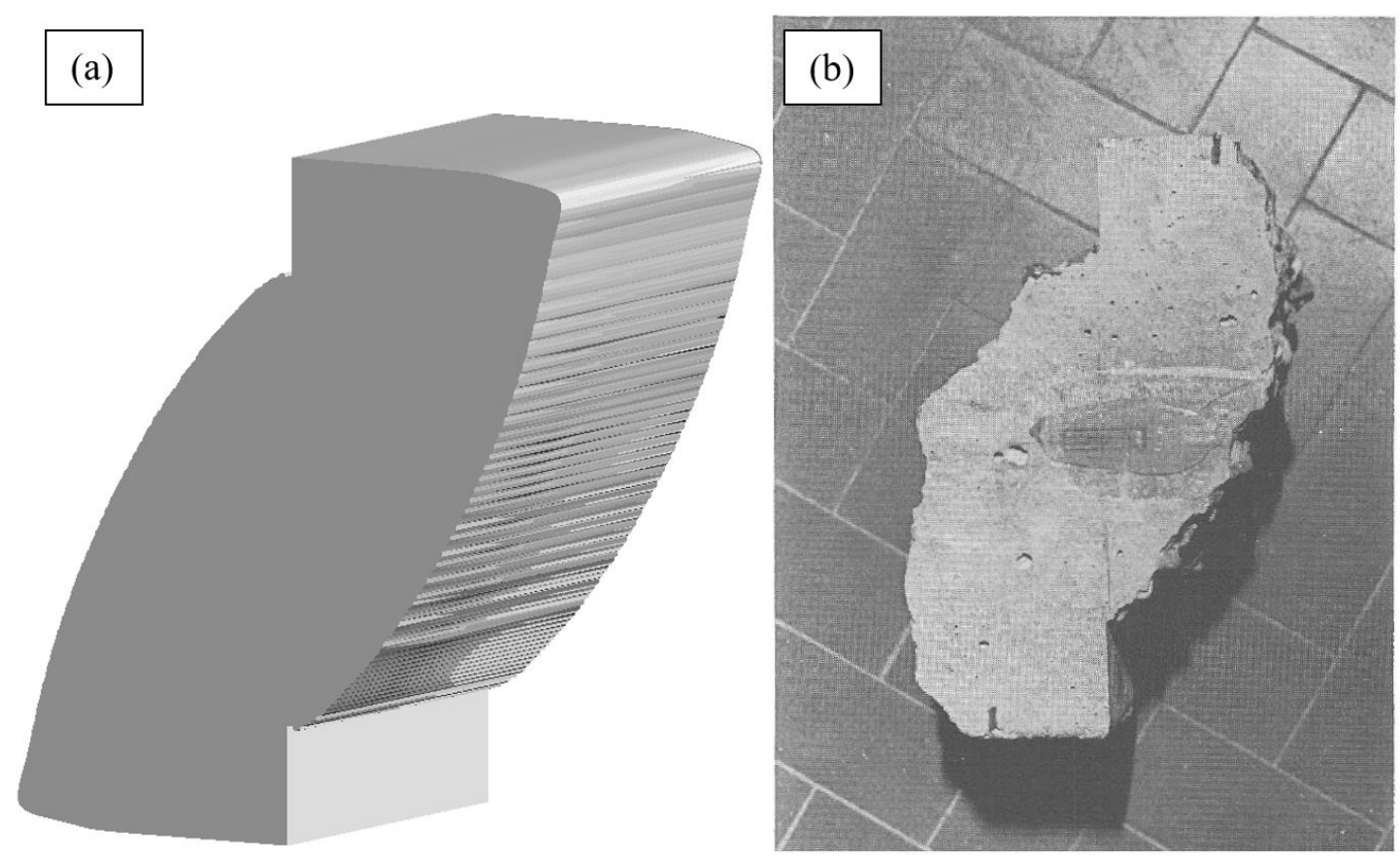

Figure 33. Central piece of (a) the modelled crack surfaces for the four-point bending test on a doubly notched beam and (b) a similar experiment [74]

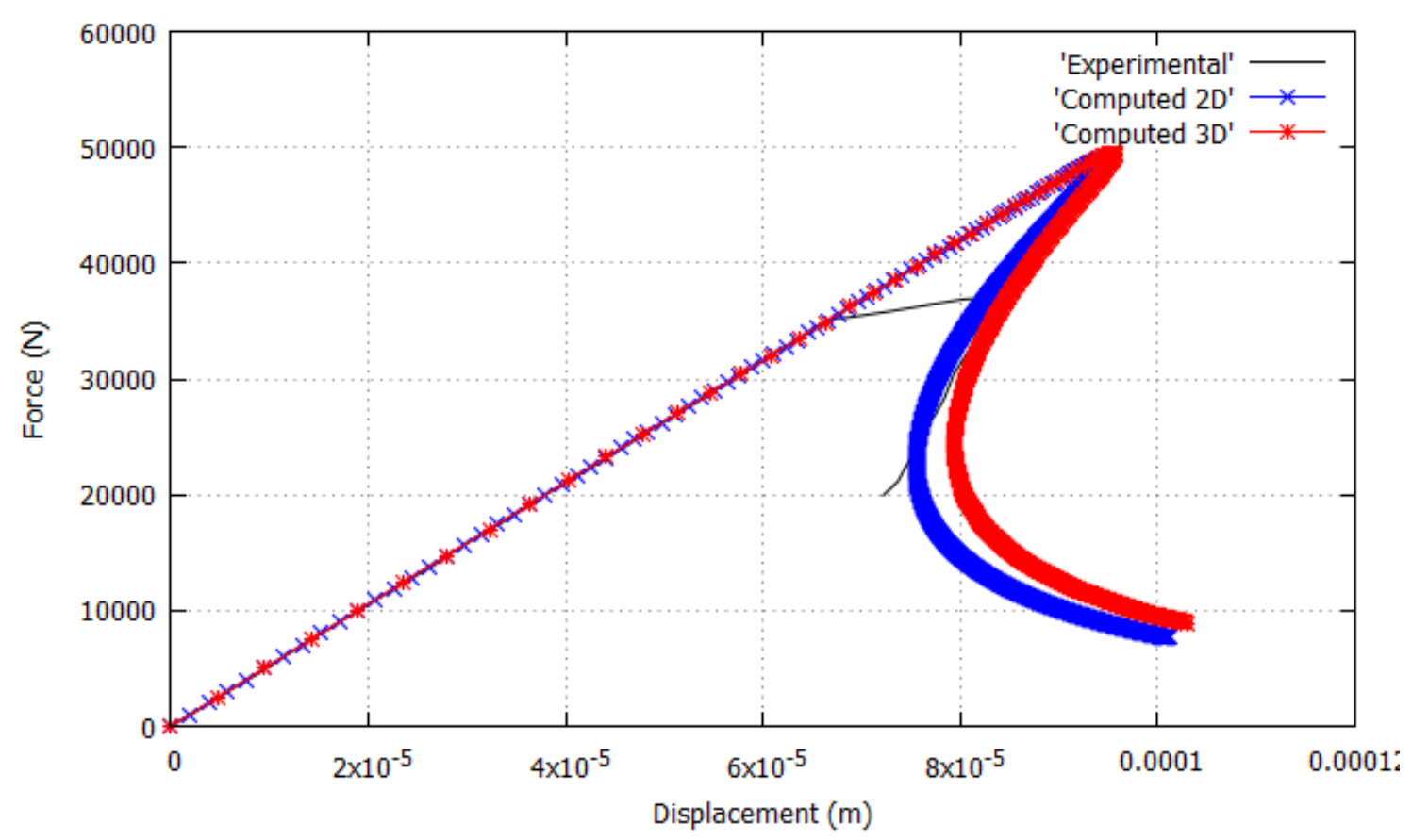

Figure 34. Force-displacement curve of the 2D and 3D simulations of the four-point bending test on a doubly notched beam 
In Figure 34, the Force-displacement curve at the point of load application is shown for the $2 \mathrm{D}$ and $3 \mathrm{D}$ computations. The computed results show very good agreement with the experimental ones of reference [74], and also with the numerical results in [37, 76].

Figure 35 shows the crack propagation and evolution of major principal stresses in the doubly-notched beam. Again it can be seen how in the elastic range stresses concentrate at the vicinity of the crack, causing its propagation through the height of the beam and towards the points of application of the loads, as seen in Figures 30, 31 and 34a. Note that the stress field is polar symmetric in the linear and in the nonlinear behavior.
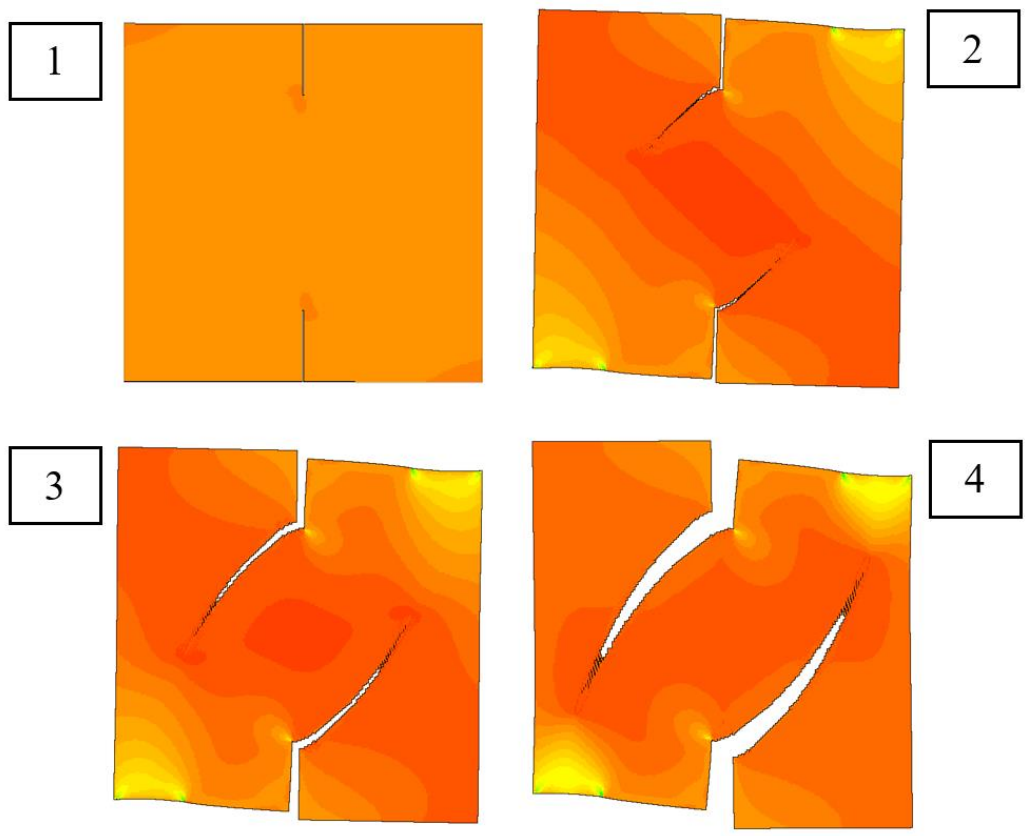

Figure 35. Crack propagation and evolution of major principal stresses in the four-point bending test on a doubly notched beam

\subsection{Three-point bending test on a skew notched beam}

In this section, a skew notched beam subjected to three-point bending is considered. The experimental tests were carried out by [77] using Plexiglass, to better reveal the evolution of the crack. Other numerical results are reported in [78], where a dual boundary element method (DBEM) was implemented and in [79], where the extended finite element method (XFEM) was used.

In Figure 36 the geometry of the tested beam is shown. The notch has a deviation of $45^{\circ}$ with respect to the lateral faces of the beam. The thickness of the specimen is $0.01 \mathrm{~m}$. The properties of the material are given in Table 6 . The structural problem is skew-symmetric with respect to the vertical longitudinal and transversal mid-planes of the beam. Therefore, a non-planar crack is foreseen to materialize at the tip of the skew notch under mixed Mode I and Mode III loading and to propagate upwards while twisting around the vertical central axis until it is oriented normal to the longitudinal axis. 

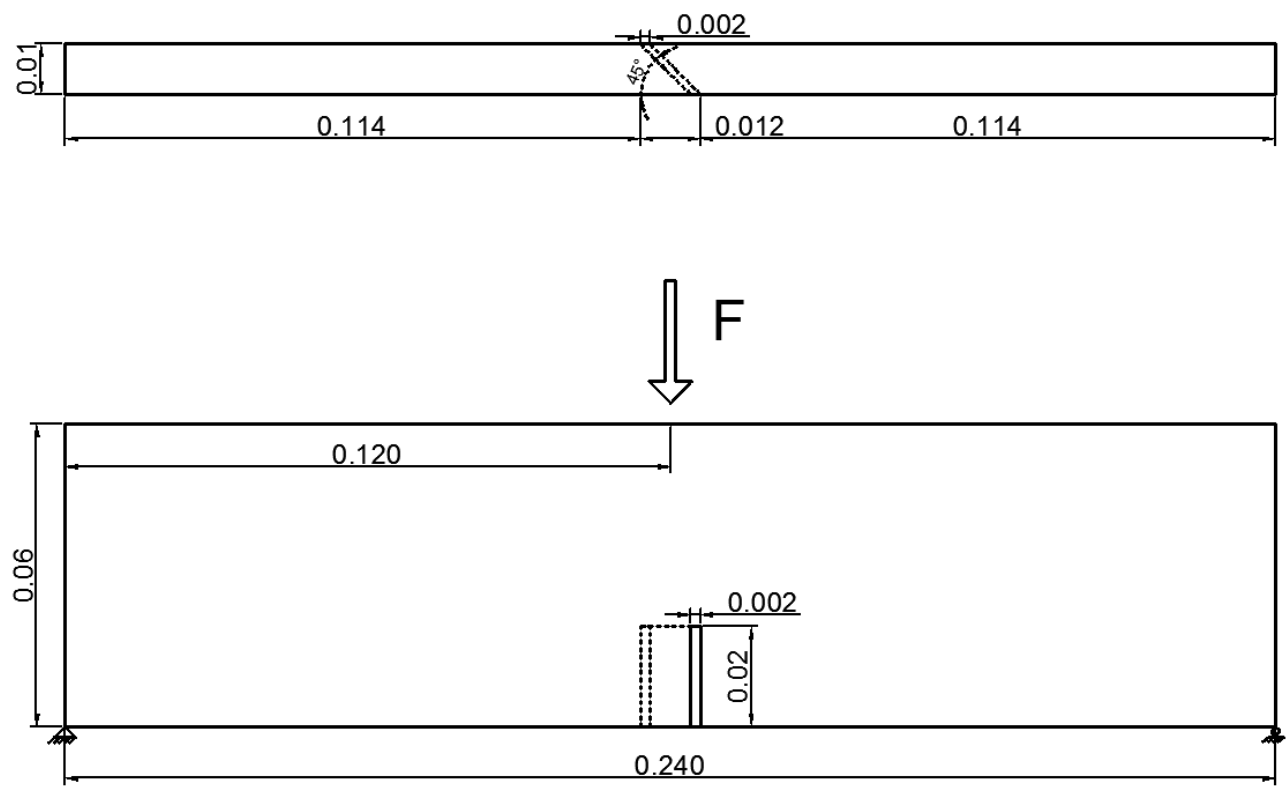

Figure 36. Geometry of the three-point bending test on a skew notched beam (m)

Table 6. Material properties of the three-point bending test on a skew notched beam

\begin{tabular}{|c|c|}
\hline Young's Modulus & $28 \cdot 10^{9} \mathrm{~Pa}$ \\
\hline Poisson's Ratio & 0.38 \\
\hline Tensile Strength & $40.0 \cdot 10^{6} \mathrm{~Pa}$ \\
\hline Fracture Energy & $500 \mathrm{~J} / \mathrm{m}^{2}$ \\
\hline
\end{tabular}
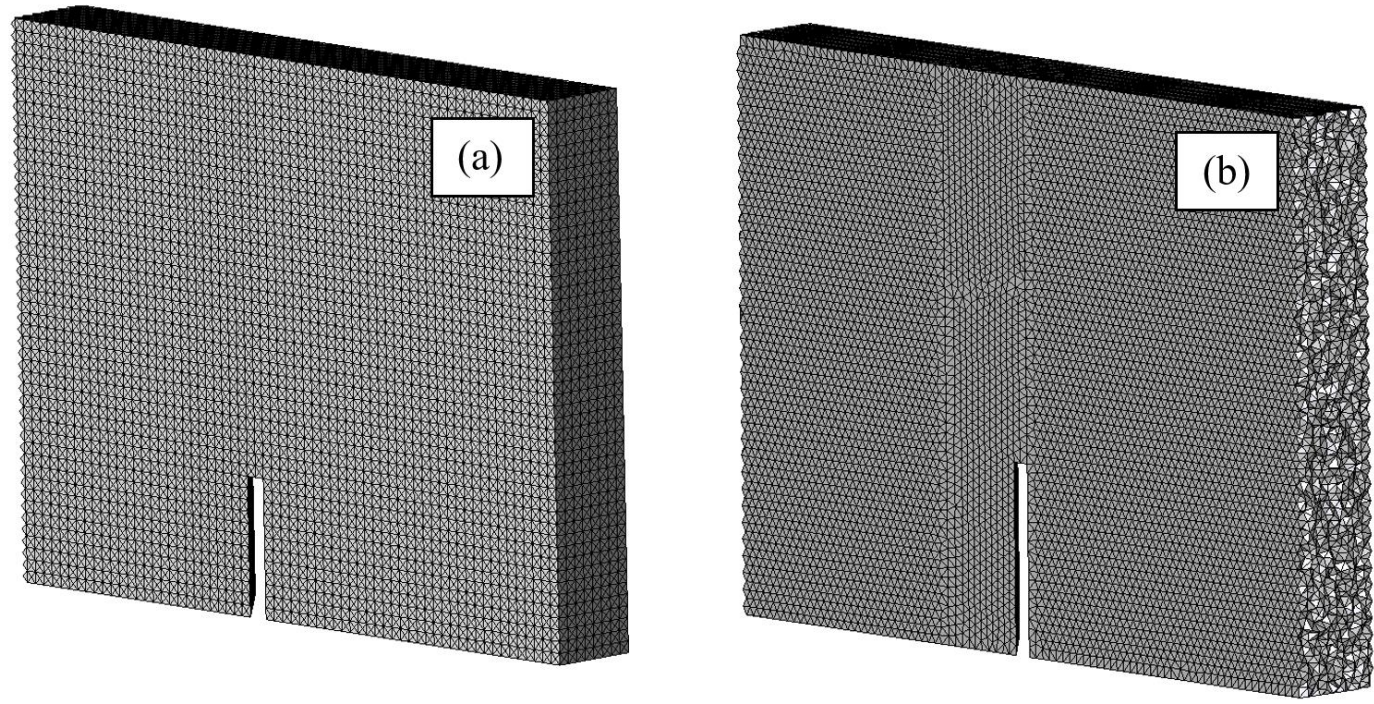

Figure 37. Meshes used for the (a) structured and (b) unstructured analyses of the three-point bending test on skew notched beam

Due to the deviation of the notch, this example can only be solved in 3D. The load is applied imposing increments of displacement at the center of the top of the beam. 3D tetrahedral elements are used in a fully structured mesh of 21,516 nodes and $1.5 \mathrm{~mm}$ 
element size in the central area of interest. The elements of the structured mesh are laid out in a crisscross pattern. Another mesh, fully unstructured, of 14,709 nodes and $1 \mathrm{~mm}$ element size is also considered. Both meshes are shown in Figure 37.
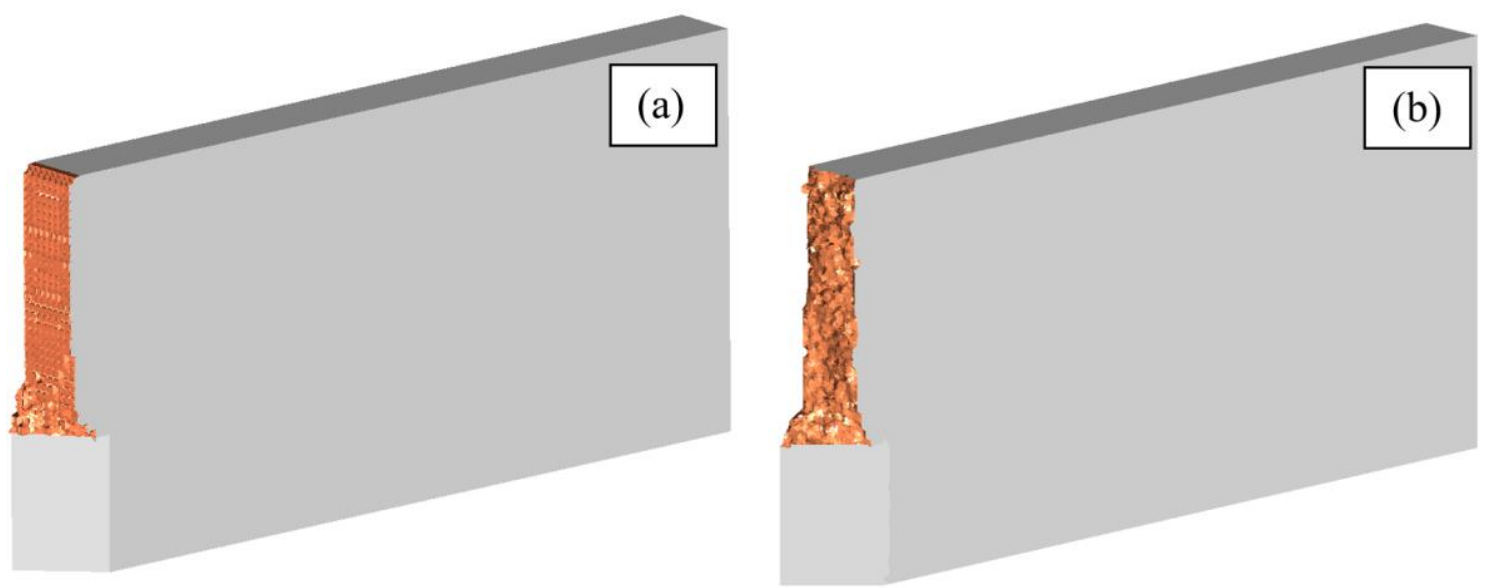

Figure 38. Crack surfaces of the three-point bending test on skew notched beam for (a) structured and (b) unstructured 3D tetrahedra mesh

In Figure 38 the crack surfaces are shown, plotted as an iso-level surface of the horizontal displacements along the axis of the beam. Both the structured and unstructured meshes used for the computation of the beam are fine enough to model the crack surface with precision. As can be seen in Figure 39, the numerical results agree well with the experimental results.

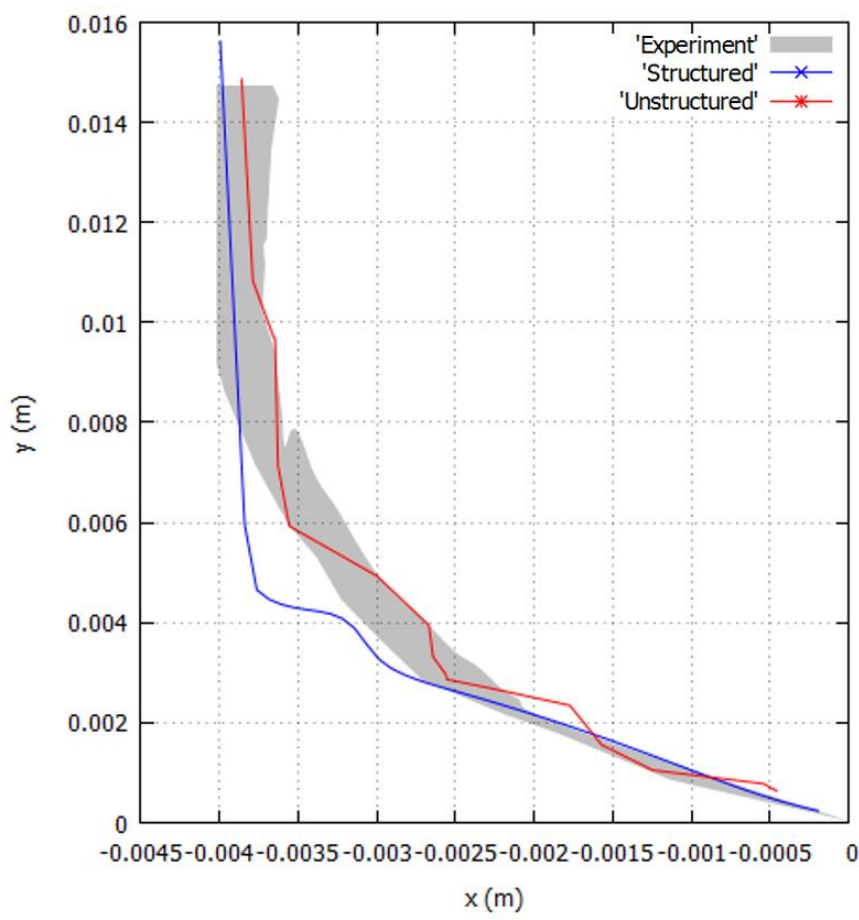

Figure 39. Crack paths of the three-point bending test on skew notched beam 


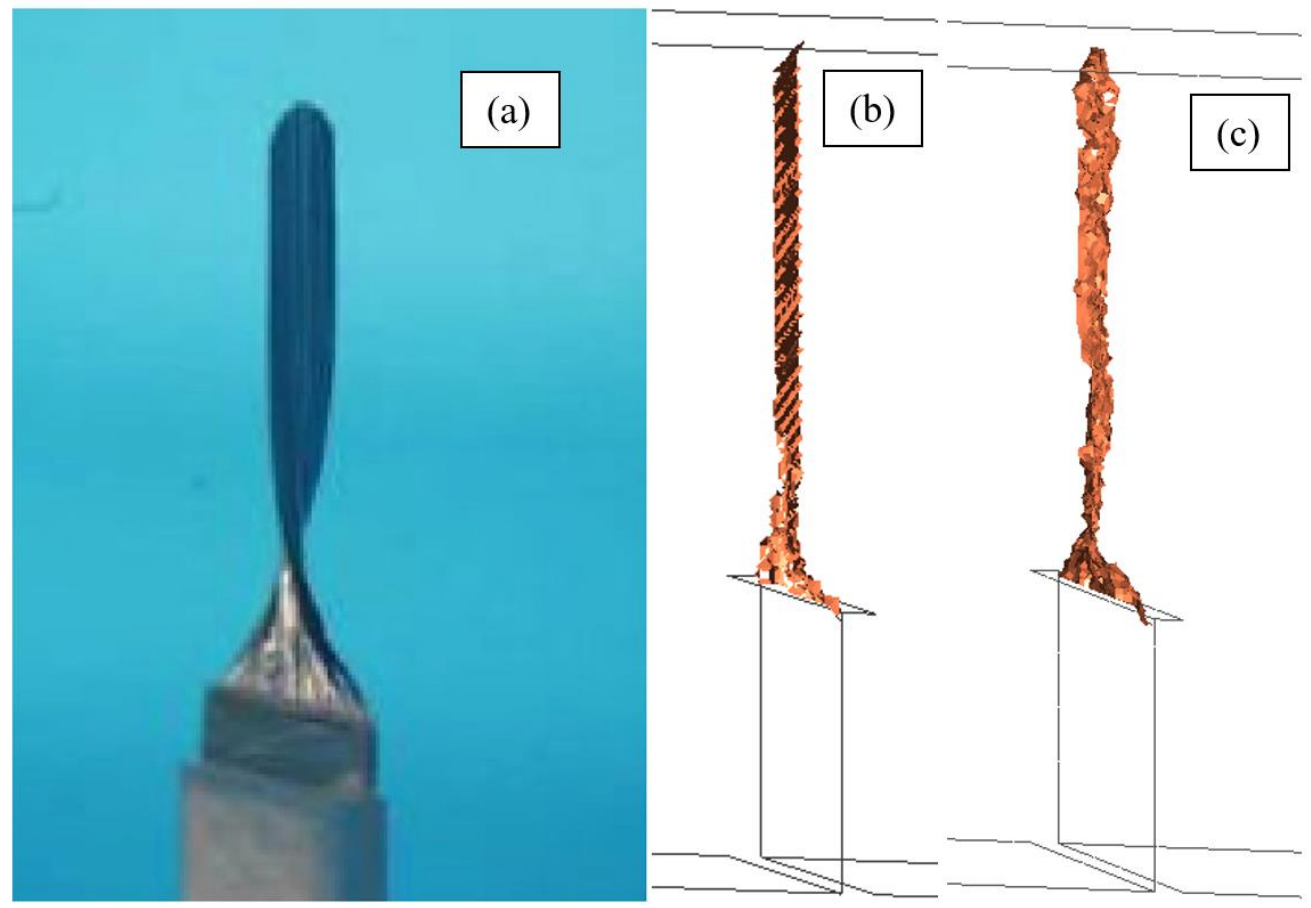

Figure 40. (a) Experimental [77] and computed crack surfaces with (b) structured and (c) unstructured meshes
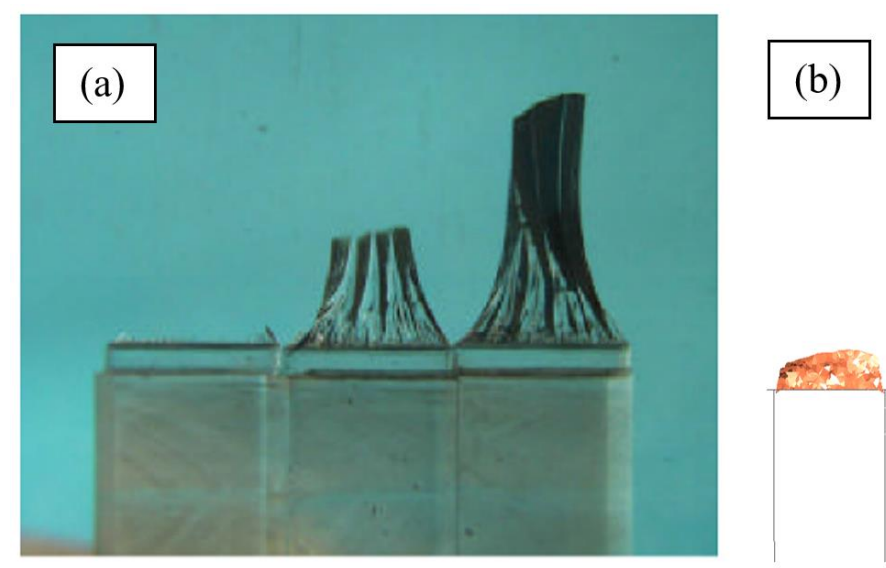

Figure 41. Evolution of the crack surface in (a) the experiment [77] and in (b) the present simulation

In Figure 40, the crack surface computed in the simulations is compared to the one observed in the tests. The results are optimal within the spatial resolution of the considered meshes. Figure 38 to Figure 40 show that results with the structured and unstructured meshes are in good agreement with experiments as well as with the skewsymmetry conditions of the problem. Evolution of the crack surface is shown in Figure 41.

Detail of the evolution of the twist angle of the crack front with the height over the initial notch is shown in Figure 42. A distinct anti-symmetric crack trace along the straight crack front can be noticed. This is because of the combination of Mode I and the antisymmetric 
Mode III loading conditions along the initial notch. The initial crack plane is inclined $45^{\circ}$ and ends at $90^{\circ}$ and only Mode I along the final crack fronts.

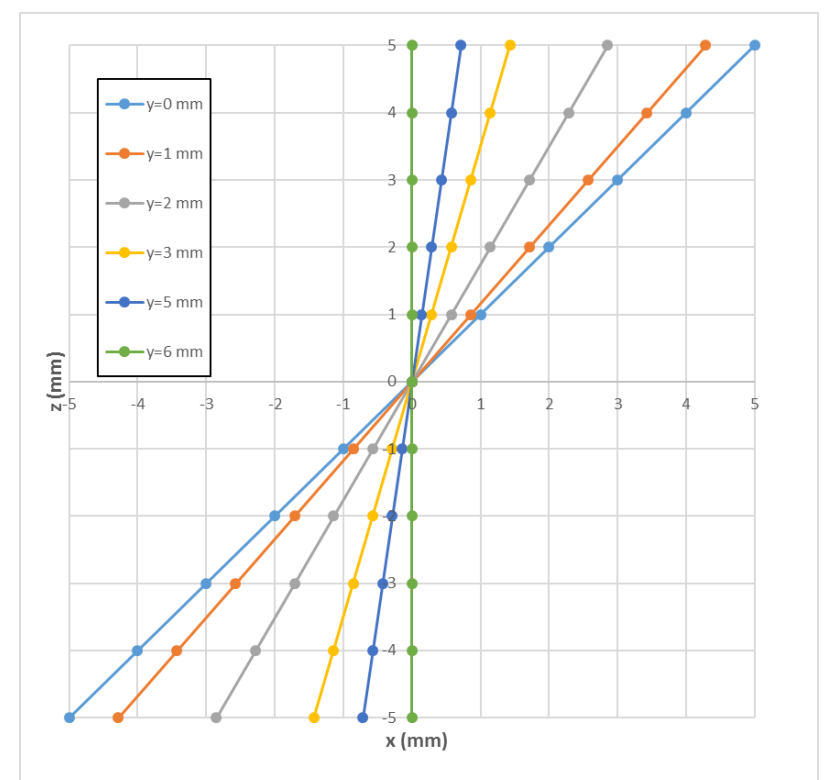

Figure 42. Computed evolution of the twist angle of the crack front with the height over the notch
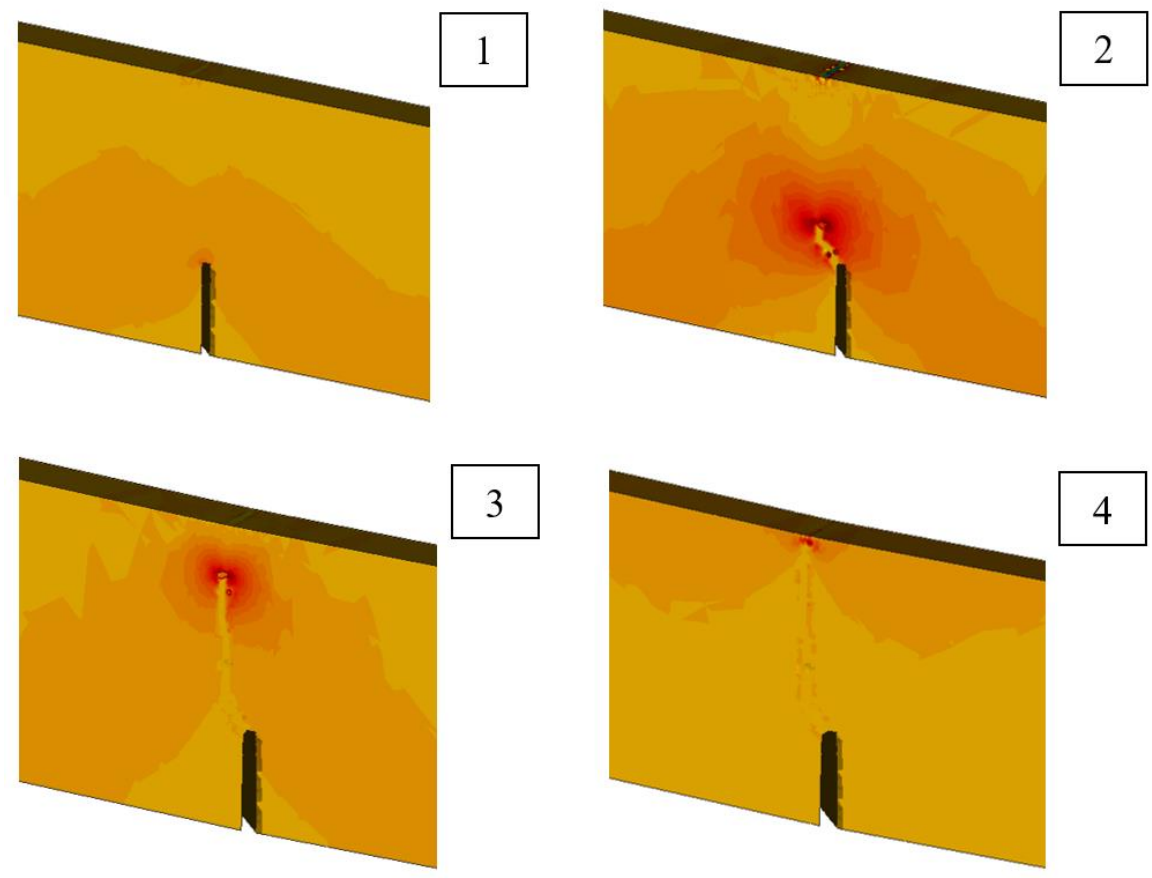

Figure 43. Crack propagation and evolution of major principal stresses in the three-point bending test on skew notched beam

Figure 43 shows the crack propagation and evolution of major principal stresses in the skew notched beam. Once more, in the elastic range, stresses concentrate in the vicinity of the tip of the notch. This causes the crack to start and propagate through the height of the beam and towards the point of application of the load. 


\section{Conclusions}

In this work, the FE modeling of quasi-brittle cracks in 2D and 3D with enhanced strain accuracy is performed. To this end, a mixed strain/displacement formulation is presented, in a matrix and vector notation, based on Voigt's convention, in a ready-to-use format for its implementation in finite element codes. Then, experimental validation is performed by means of several simulations which are compared to experimental tests reported in the literature.

The proposed formulation is used in conjunction with an isotropic damage model suitable for the prediction of cracking in 2D and 3D applications. Finite element simulations using triangles, quadrilaterals, tetrahedra, hexahedra and prisms in structured, semi-structured and unstructured meshes are performed.

An extensive comparison with experimental data observed from the literature is carried out, to assess the capacity of the proposed formulation to model the behavior observed in the experimental tests. Several numerical simulations have been exhibited to illustrate the capacity of the formulation to solve strain localization problems in accordance to experimental results.

Problems involving propagation of single and multiple, straight and curved cracks in 2D and 3D, as well as non-planar cracks in 3D are addressed. Aspects related to the discrete solution, such as convergence regarding mesh resolution and mesh bias, as well as other related to the physical model, like structural size effect and the influence of Poisson's ratio, are also investigated.

The enhanced accuracy of the computed strain field leads to accurate results in terms of crack paths, failure mechanisms and force displacement curves. Spurious mesh dependency suffered by both continuous and discontinuous irreducible formulations is avoided by the mixed FE, without the need of auxiliary tracking techniques or other computational schemes that alter the continuum mechanical problem.

\section{References}

[1] Y. Rashid, "Ultimate strength analysis of prestressed concrete pressure vessels," Nuclear Engineering and Design, vol. 7, no. 4, pp. 334-344, 1968.

[2] Z. Bazant and B. Oh, "Crack band theory for fracture of concrete," Matériaux et Constructions, vol. 16, no. 3, pp. 155-177, 1983.

[3] J. Rots, P. Nauta and G. Kusters, "Variable reduction factor for the shear stiffness of cracked concrete," Rep. BI-84-33, Ins. TNO for building Mat. And Struct., Delft, 1984. 
[4] R. de Borst and P. Nauta, "Non-orthogonal cracks in a smeared finite element model," Engng. Computation, vol. 2, pp. 35-46, 1985.

[5] R. de Borst, "Smeared cracking, plasticity, creep and thermal loading - a unified approach," Comp. Meth. Appl. Mech. Eng., vol. 62, no. 99, pp. 89-110, 1987.

[6] Z. Bazant and G. Pijaudier-Cabot, "Nonlocal continum damage, localization instabilities and convergence," Journal of Engineering Mechanics , no. 55, pp. 287293, 1988.

[7] R. Peerlings, R. de Borst, W. Brekelmans and J. de Wree, "Gradient enhanced damage for quasi brittle materials," International Journal for Numerical Methods in Engineering, vol. 39, pp. 3391-3403, 1996.

[8] R. de Borst and C. Verhoosel, "Gradient damage vs phase-field approaches for fracture: Similarities and differences.," Comput. Methods Appl. Mech. Engrg., p. doi: 10.1016/j.cma.2016.05.015, 2016.

[9] C. Miehe, F. Welschinger and M. Hofacker, "Thermodynamically consistent phasefield models of fracture: Variational principles and multi-field FE implementations," International Journal for Numerical Methods in Engineering, vol. 83, pp. 1273-1311, 2010.

[10] C. Miehe, L.-M. Schänzel and H. Ulmer, "Phase field modeling of fracture in multiphysics problems. Part I. Balance of cracks surface and failure criteria for brittle crack propagation in thermo-elastic solids," Computer Methods in Applied Mechanics and Engineering, vol. 294, pp. 449-485, 2015.

[11] J. Vignollet, S. May, R. de Borst and C. Verhoosel, "Phase-field model for brittle and cohesive fracture," Meccanica, vol. 49, pp. 2587-2601, 2014.

[12] J. Wu, "A geometrically regularized gradient-damage model with energetic equivalence," submitted to Computer Methods in Applied Mechanics and Engineering, 2017.

[13] G. Barenblatt, "The mathematical theory of equilibrium cracks in brittle faillure," Advanced Applied Mathematics, vol. 7, pp. 55-129, 1962.

[14] D. Ngo and A. Scordelis, "Finite element analysis of reinforced concrete beams," ACI Journal, vol. 64, no. 14, pp. 152-163, 1967.

[15] P. Areias, M. Msekh and T. Rabczuk, "Damage and fracture algorithm using the screened Poisson equation and local remeshing," Engineering Fracture Mechanics, vol. 158, pp. 116-143, 2016.

[16] P. Areias and T. Rabczuk, "Finite strain fracture of plates and shells with configurational forces and edge rotations," International Journal for Numerical Methods in Engineering, vol. 94, pp. 1099-1122, 2013. 
[17] P. Areias, T. Rabczuk and D. Dias-da-Costa, "Element-wise fracture algorithm based on rotation of edges," Engineering fracture mechanics, vol. 110, pp. 113-137, 2013.

[18] P. Areias, J. Reinoso, P. Camanho and T. Rabczuk, "A constitutive-based elementby-element crack propagation algorithm with local mesh refinement," Computational Mechanics, vol. 56, pp. 291-315, 2015.

[19] J. Schellekens, "A non-linear finite element approach for the analysis of mode-I free edge delamination in composites," International Journal of Solids and Structures, vol. 30, no. 9, pp. 1239-1253, 1993.

[20] O. Allix and P. Ladevèze, "Interlaminar interface modelling for the prediction of delamination," Composite Structures, vol. 22, no. 4, pp. 235-242, 1992.

[21] G. Bolzon and A. Corigliano, "A discrete formulation for elastic solids with damaging interfaces," Comp. Methods Appl. Mech. Engng., vol. 140, pp. 329-359, 1997.

[22] A. Pandolfi, P. Krysl and M. Ortiz, "Finite element simulation of ring expansion and fragmentation: The capturing of length and time scales through cohesive models of fracture," International Journal of Fracture, vol. 95, no. 1-4, pp. 279297, 1999.

[23] E. Dvorkin, A. Cuitino and G. Gioia, "Finite elements with displacement interpolated embedded localization lines insensitive to mesh size and distorsions," International Journal for Numerical Methods in Engineering, vol. 30, pp. 541-564, 1990.

[24] J. Oliver, M. Cervera and O. Manzoli, "Strong discontinuities and continuum plasticity models: the strong discontinuity approach," International Journal of Plasticity, vol. 15, no. 3, pp. 319-351, 1999.

[25] T. Gasser and G. Holzapfel, "Geometrically non-linear and consistently linearized embedded strong discontinuity models for 3D problems with an application to the dissection analysis of soft biological tissues," Computer Methods in Applied Mechanics and Engineering, vol. 192, pp. 5059-5098, 2003.

[26] M. Motamedi, D. Weed and C. Foster, "Numerical simulation of mixed mode (I and II) fracture behaviour pf pre-cracked rock using the strong discontinuity approach," International Journal of Solids and Structures, Vols. 85-86, pp. 44-56, 2016.

[27] Y. Zhang, R. Lackner, M. Zeiml and H. Mang, "Strong discontinuity embedded approach with standard SOS formulation: Element formulation, energy-based crack tracking strategy, and validations," Computer Methods in Applied Mechanics and Engineering, vol. 287, pp. 335-366, 2015. 
[28] X. Su, Z. Yang and G. Liu, "Finite element modelling of complex 3D static and dynamic crack propagation by embedding cohesive elements in Abaqus," Acta Mecanica Solida Sinica, vol. 23, no. 3, pp. 271-282, June 2010.

[29] N. Moes, J. Dolbow and T. Belytschko, "A finite element method for crack growth without remeshing," International Journal for Numerical Methods in Engineering, vol. 46, no. 1, pp. 131-150, 1999.

[30] T. Gasser and G. Holzapfel, "Modeling 3D crack propagation in unreinforced concrete using PUFEM," Computer Methods in Applied Mechanics and Engineering, vol. 194, pp. 2859-2896, 2005.

[31] M. Holl, T. Rogge, S. Loehnert, P. Wriggers and R. Rolfes, "3D multiscale crack propagation using the XFEM applied to a gas turbine blade," Computational Mechanics, vol. 53, pp. 173-188, 2014.

[32] G. Meschke and P. Dumstorff, "Energy-based modeling of cohesive and cohesionless cracks via X-FEM," Computer Methods in Applied Mechanics and Engineering, vol. 196, pp. 2338-2357, 2007.

[33] J.-Y. Wu and F.-b. Li, "An improved stable X-FEM (Is-FEM) with a novel enrichment function for the computational modeling of cohesive cracks," Computer Methods in Applied Mechanics and Engineering, vol. 295, pp. 77-107, 2015.

[34] P. Areias and T. Belytschko, "Analysis of three-dimensional crack initiation and propagation using the extended finite element method," Int. J. Numer. Meth. Engng., vol. 63, pp. 760-788, 2005.

[35] T. Rabczuk and T. Belytschko, "A three-dimensional large deformation meshfree method for arbitrary evolving cracks," Computer Methods in Applied Mechanics and Engineering, vol. 196, pp. 2777-2799, 2007.

[36] T. Rabczuk and T. Belytschko, "Adaptivity for structured meshfree particle methods in 2D and 3D," International Journal for Numerical Methods in Engineering, vol. 63, pp. 1559-1582, 2005.

[37] T. Rabczuk and T. Belytschko, "Cracking particles: a simplified meshfree method for arbitrary evolving cracks," International Journal for Numerical Methods in Engineering, vol. 61, pp. 2316-2343, 2004.

[38] X. Zhuang, C. Augarde and S. Bordas, "Accurate fracture modelling using meshless methods, the visibility criterion and level sets: Formulation and 2D modelling," International Journal for Numerical Methods in Engineering, vol. 86, pp. 249-268, 2011.

[39] X. Zhuang, C. Augarde and K. Mathisen, "Fracure modeling using meshless methods and level sets in 3D: framework and modeling," International Journal for Numerical Methods in Engineering, vol. 92, pp. 969-998, 2012. 
[40] G. Nguyen, C. Nguyen, P. Nguyen, H. Bui and L. Shen, "A size-dependent constitutive modelling framework for localized faillure analysis," Comput. Mech., vol. 58, pp. 257-280, DOI 10.1007/s00466-016-1293-z, 2016.

[41] C. Annavarapu, R. Settgast, E. Vitali and J. Morris, "A local crack-tracking strategy to model three-dimensional crack propagation with embedded methods," Comput. Methods Appl. Mech. Engrg., vol. 311, pp. 815-837, 2016.

[42] P. Dumstorffz and G. Meschke, "Crack propagation criteria in the framework of XFEM-based structural analysis," Int. J. Numer. Anal. Meth. Geomech., vol. 31, pp. 239-259, 2007.

[43] J. Kim and F. Armero, "Three-dimensional finite elements with embedded strong discontinuities for the analysis of solids at faillure in the finite deformation range," Comput. Methods Appl. Mech. Engrg., no. http://dx.doi.org/10.1016/j.cma.2016.12.038, 2017.

[44] F. Riccardi, E. Kishta and B. Richard, "A step-by-step global crack-tracking approach in E-FEM simulations of quasi-brittle materials," Engineering Fracture Mechanics, vol. 170, pp. 44-58, 2017.

[45] D. Dias-da- Costa, J. Alfaiate, L. Sluys and Júlio E., "A comparative study on the modelling of discontinuous fracture by means of enriched nodal and element techniques and interface elements," Int. J. Fract., vol. 161, no. 1, pp. 97-119, 2010.

[46] M. Jirasek, "Comparative study on finite elements with embedded discontinuities," Comput. Methods Appl. Mech. Eng., vol. 188, pp. 307-330, 2000.

[47] T. Rabczuk, "Computational methods for fracture in brittle and quasi-brittle solids: state-of-the-art review and future perspectives," ISRN Appl. Math. 2013, p. DOI: 10.1155/2013/849231, 2013.

[48] M. Cervera, M. Chiumenti and R. Codina, "Mesh objective modelling of cracks using continuous linear strain and displacement interpolations," International Journal for Numerical Methods in Engineering, vol. 87, no. 10, pp. 962-987, 2011.

[49] M. Cervera, M. Chiumenti and R. Codina, "Mixed stabilized finite element methods in nonlinear solid mechanics. Part I: Formulation," Computer Methods in Applied Mechanics and Engineering, vol. 199, no. 37-40, pp. 2559-2570, 2010.

[50] M. Cervera, M. Chiumenti and R. Codina, "Mixed stabilized finite element methods in nonlinear solid mechanics. Part II: Strain localization," Computer Methods in Applied Mechanics and Engineering, vol. 199, no. 37-40, pp. 2571-2589, 2010.

[51] M. Cervera, M. Chiumenti, L. Benedetti and R. Codina, "Mixed stabilized finite element methods in nonlinear solid mechanics. Part III: Compressible and incompressible plasticity," Computer Methods in Applied Mechanics and Engineering, vol. 285, no. 0, pp. 752-775, 2015. 
[52] A. Gil, C. Lee, J. Bonet and M. Aguirre, "A stabilized Petrov-Galerkin formulation for linear tetrahedral elements in compressible, nearly incompressible and truly incompressible fast dynamics," Comput. Methods Appl. Mech. Engrg., vol. 276, pp. 659-690, 2014.

[53] N. Lafontaine, R. Rossi, M. Cervera and M. Chiumenti, "Explicit mixed straindisplacement finite element for dynamic geometrically non-linear solid mechanics," Computational Mechanics, vol. 55, pp. 543-559, 2015.

[54] M. Cervera and M. Chiumenti, "Size effect and localization in J2 plasticity," International Journal of Solinds and Structures, vol. 46, pp. 3301-3312, 2009.

[55] L. Benedetti, M. Cervera and M. Chiumenti, "3D modelling of twisting cracks under bending and torsion skew notched beams," Engineering Fracture Mechanics, vol. 176, pp. 235-256, 2017.

[56] M. Chiumenti, M. Cervera and R. Codina, "A mixed three-field FE formulation for stress accurate analysis including the incompressible limit," Comput. Methods Appl. Mech. Engrg., vol. 283, pp. 1095-1116, 2014.

[57] E. Hellinger, "Die allegemeinen Ansätze der Mechanik der Kontinua, Art. 30," Encyclopädie der Matematischen Wissenschaften, F. Klein and C. Muller (eds.), Leipzig, Teubner, pp. 654-655, 1914.

[58] E. Reissner, "On variational principles of elasticity," Proc. Symp. Appl. Math., vol. 8, pp. 1-6, 1958.

[59] O. Zienkiewicz, R. Taylor and Z. Zhu, "The finite element method, Vol. 1," 7th edition, Amsterdam, Elsevier Butterworth-Heinemann, 1989.

[60] I. Babuska, "Error-bounds for finite element method," Numerisch Mathematik, vol. 16, pp. 322-333, 1971.

[61] D. Boffi, F. Brezzi and M. Fortin, "Mixed Finite Element Methods and Applications," Springer, 2013.

[62] F. Brezzi, "On the existence, uniqueness and approximation of saddle-point problems arising from lagrangian multipliers," ESAIM: Mathematical Modelling and Numerical Analysis - Modélisation Mathématique et Analyse Numérique, vol. 8, no. R2, pp. 129-151, 1974.

[63] R. Codina, "Stabilization of incompressibility and convection through orthogonal sub-scales in finite element methods," Computer Methods in Applied Mechanics and Engineering , vol. 190, pp. 1579-1599, 2000.

[64] T. Hughes, G. Feijoo, L. Mazzei and J. Quincy, "The variational multiscale method - a paradigm for computational mechanics," Computer Methods in Applied Mechanics and Engineering, vol. 166, pp. 3-24, 1998. 
[65] T. Hughes, L. Franca and M. Balestra, "A new finite element formulation for computational fluid dynamics: V. Circumventing the Babuska-Brezzi condition: A stable Petrov-Galerkin formulation of the stokes problem accomodating equal-order interpolations," Computer Methods in Applied Mechanics and Engineering, vol. 59, no. 1 , pp. 85-99, 1986.

[66] M. Cervera, C. Agelet de Saracibar and M. Chiumenti, "COMET: Coupled Mechanical and Thermal Analysis. Data Input Manuel, Version 5.0, Technical report IT-308. Available from http://www.cimne.upc.edu," 2002.

[67] "GiD: the personal pre and post-processor.," CIMNE, Technical University of Catalonia, p. <http://gid.cimne.upc.ed>, 2002.

[68] B. Winkler, "Traglastuntersuchungen von unbewehrten und bewerhrten Betonskrukturen auf der Grundlage eines objektiven Werkstoffgesetzes für Beton," Ph.D. Thesis, Universität Innsbruck, 2001.

[69] B. Trunk, "Einfluss der Bauteilgrösse auf die Bruchenergie von Beton," Aedificatio publishers, Freiburg, 2000.

[70] J. Gálvez, M. Elices, G. Guinea and J. Planas, "Mixed mode fracture of concrete under proportional and nonproportional loading," Int J. Fract., vol. 94, pp. 267-84, 1998.

[71] M. Cervera, L. Pela, R. Clemente and P. Roca, "A crack-tracking technique for localized damage in quasi-brittle materials," Engineering Fracture Mechanics, vol. 77, pp. 2431-2450, 2010.

[72] A. Ingraffea and M. Grigoriu, "Probabilistic Fracture Mechanics: A Validation Predictive Capability," Tech. rep., DTIC Document, 1990.

[73] C. Miehe and E. Gürses, "A robust algorithm for the configurational-force-driven brittle crack propagation with R-adaptative mesh alignment," Int. J. Numer. Meth. Engng., vol. 72, pp. 127-155, 2007.

[74] P. Bocca, A. Carpinteri and S. Valente, "Mixed mode fracture of concrete," Int. J. Solids Structures, vol. 27, no. 9, pp. 1139-1153, 1991.

[75] A. Saleh and M. Aliabadi, "Crack growth analysis in concrete using boundary element method," Engineering Fracture Mechanics, vol. 51, no. 4, pp. 533-545, 1995.

[76] P. Areias, T. Rabczuk and J. César de Sá, "A novel two-stage discrete crack method based on the screened Poisson equation and local mesh refinement," Comput. Mech., vol. 58, pp. 1003-1018, 2016.

[77] F. Buchholz, A. Chergui and H. Richard, "Fracture analyses and experimental results of crack growth under general mixed mode loading conditions," Engineering Fracture Mechanics, vol. 71, pp. 455-468, 2004. 
[78] R. Citarella and F. Buchholz, "Comparison of crack growth simulation by DBEM and FEM for SEN-specimens undergoing torsion or bending loading," Engineering Fracture Mechanics, vol. 75, pp. 489-509, 2008.

[79] G. Ferte, P. Massin and N. Moës, "3D crack propagation with cohesive elements in the extended finite element method," Comput. Methods Appl. Mech. Engrg., vol. 300, pp. 347-374, 2016. 